\title{
Online Quantitative Timed Pattern Matching with Semiring-Valued Weighted Automata ${ }^{\star}$
}

\author{
Masaki Waga ${ }^{1,2,3[0000-0001-9360-7490]}$ \\ 1 National Institute of Informatics, Tokyo, Japan \\ 2 SOKENDAI (The Graduate University for Advanced Studies), Tokyo, Japan \\ 3 JSPS Research Fellow, Tokyo, Japan
}

\begin{abstract}
Monitoring of a signal plays an essential role in the runtime verification of cyber-physical systems. Qualitative timed pattern matching is one of the mathematical formulations of monitoring, which gives a Boolean verdict for each sub-signal according to the satisfaction of the given specification. There are two orthogonal directions of extension of the qualitative timed pattern matching. One direction on the result is quantitative: what engineers want is often not a qualitative verdict but the quantitative measurement of the satisfaction of the specification. The other direction on the algorithm is online checking: the monitor returns some verdicts before obtaining the entire signal, which enables to monitor a running system. It is desired from application viewpoints. In this paper, we conduct these two extensions, taking an automata-based approach. This is the first quantitative and online timed pattern matching algorithm to the best of our knowledge. More specifically, we employ what we call timed symbolic weighted automata to specify quantitative specifications to be monitored, and we obtain an online algorithm using the shortest distance of a weighted variant of the zone graph and dynamic programming. Moreover, our problem setting is semiring-based and therefore, general. Our experimental results confirm the scalability of our algorithm for specifications with a time-bound.
\end{abstract}

Keywords: quantitative monitoring, timed automata, weighted automata, signals, zones, dynamic programming, semirings, timed pattern matching, runtime verification

\section{Introduction}

Background Monitoring a system behavior plays an essential role in the runtime verification or falsification of cyber-physical systems (CPSs), where various for-

\footnotetext{
* This is the author (and extended) version of the manuscript of the same name published in the proceedings of the 17th International Conference on Formal Modeling and Analysis of Timed Systems (FORMATS 2019). The final version is available at www. springer. com. This version contains additional proofs. Thanks are due to Ichiro Hasuo for a lot of useful comments and Sasinee Pruekprasert for a feedback. This work is partially supported by JST ERATO HASUO Metamathematics for Systems Design Project (No. JPMJER1603) and by JSPS Grants-in-Aid No. 15 KT0012 \& 18J22498.
} 
Table 1: Comparison of the problem settings with related studies

\begin{tabular}{c||c|c|c|c} 
& Quantitative? & Online? & Dense time? & Result of which part? \\
\hline \hline$\left[\right.$ BFN $\left.^{+} 18\right]$ & No & Yes & Yes & All sub-signals (pattern matching) \\
\hline$[$ BFMU17] & Yes & No & Yes & All sub-signals (pattern matching) \\
\hline$\left[\mathrm{JBGN}_{18}\right]$ & Yes & Yes & No & The whole signal \\
\hline$\left[\mathrm{DDG}^{+} 15\right]$ & Yes & Yes & Yes & The whole signal \\
\hline This Paper & Yes & Yes & Yes & All sub-signals (pattern matching)
\end{tabular}

malisms such as temporal logic formulas or automata are used for specification. Usually, a CPS is a real-time system, and real-time constraints must be included in the specification. An example of such a specification is that the velocity of a self-driving car should be more than $70 \mathrm{~km} / \mathrm{h}$ within $3 \mathrm{~s}$ after the car enters an empty highway. Timed automata [AD94] is a formalism that captures realtime constraints. They are equipped with clock variables and timing constraints on the transitions. Applications of monitoring of real-time properties include data classification $\left[\mathrm{BVP}^{+} 16\right]$ and Web services [RSE08] as well as CPSs (e.g., automotive systems [KCDK15] and medical systems [CSWL16]).

The behavior of a CPS is usually described as a real-valued signal that is mathematically a function $\sigma$ mapping a time $t$ to the condition $\sigma(t) \in \mathbb{R}^{n}$ of the system at time $t$. Usual automata notions (e.g., NFA and timed automata) handle only finite alphabets, and in order to monitor signals over $\mathbb{R}^{n}$, automata must be extended to handle infinite alphabets. Symbolic automata $\left[\mathrm{VHL}^{+} 12\right]$ handle large or even infinite alphabets, including real vectors. In a symbolic automaton over a real vector space $\mathbb{R}^{n}$, each location (or transition) is labeled with a constraint over $\mathbb{R}^{n}$ instead of one vector $v \in \mathbb{R}^{n}$; therefore, one location (or transition) corresponds to infinitely many vectors.

Monitoring can be formulated in various ways. They are classified according to the following criteria. Table 1 shows a comparison of various formulations of monitoring problems.

Qualitative vs. quantitative semantics When an alphabet admits subtraction and comparisons, in addition to the qualitative semantics (i.e., true or false), one can define a quantitative semantics (e.g., robustness) of a signal with respect to the specification [FP09,DM10,AH15,BFMU17]. Robust semantics shows how robustly a signal satisfies (or violates) the given specification. For instance, the specification $v>70$ is satisfied more robustly by $v=170$ than by $v=70.0001$. In the context of CPSs, robust semantics for signal temporal logic is used in robustness-guided falsification [Don10,ALFS11]. Weighted automata [Sch61,DKV09] are employed for expressing such a quantitative semantics [ $\mathrm{JBG}^{+} 18, \mathrm{JBGN18}$.

Offline vs. online Consider monitoring of a signal $\sigma=\sigma_{1} \cdot \sigma_{2}$ over a specification $\mathcal{W}$. In offline monitoring, the monitor returns the result $\mathcal{M}(\sigma, \mathcal{W})$ after obtaining the entire signal $\sigma$. In contrast, in online monitoring, the monitor starts returning the result before obtaining the entire signal $\sigma$. For example, the monitor may return a partial result $\mathcal{M}\left(\sigma_{1}, \mathcal{W}\right)$ for the first part $\sigma_{1}$ before obtaining the second part $\sigma_{2}$. 

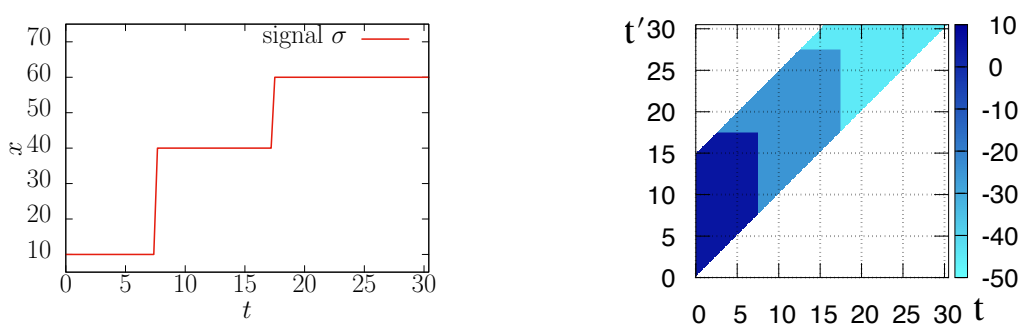

Fig. 1: Piecewise-constant signal $\sigma$ (left) and an illustration of the quantitative matching function $(\mathcal{M}(\sigma, \mathcal{W}))\left(t, t^{\prime}\right)$ for $\left[t, t^{\prime}\right) \subseteq[0,30.5)$ (right). In the right figure, the score in the white areas is $-\infty$. The specification $\mathcal{W}$ is outlined in Example 1. In the right figure, the value at $(3,15)$ is 5 . It shows that the score $(\mathcal{M}(\sigma, \mathcal{W}))(3,15)$, for the restriction $\sigma([3,15))$ of $\sigma$ to the interval $[3,15)$, is 5 .

Discrete vs. dense time In a discrete time setting, timestamps are natural numbers while, in a dense time setting, timestamps are positive (or nonnegative) real numbers.

Result of which part? Given a signal $\sigma$, we may be interested in the properties of different sets of sub-signals of $\sigma$. The simplest setting is where we are interested only in the whole signal $\sigma$ (e.g., $\left.\left[\mathrm{DDG}^{+} 15, \mathrm{JBGN} 18\right]\right)$. Another more comprehensive setting is where we are interested in the property of each sub-signal of $\sigma$; problems in this setting are called timed pattern matching [UFAM14,WAH16,BFMU17].

Our problem Among the various problem settings of monitoring, we focus on an online algorithm for quantitative timed pattern matching [BFMU17] in a dense time setting. See Table 1. Given a piecewise-constant signal $\sigma$ and a specification $\mathcal{W}$ expressed by what we call a timed symbolic weighted automaton, our algorithm returns the quantitative matching function $\mathcal{M}(\sigma, \mathcal{W})$ that maps each interval $\left[t, t^{\prime}\right) \subseteq[0,|\sigma|)$ to the (quantitative) semantics $(\mathcal{M}(\sigma, \mathcal{W}))\left(t, t^{\prime}\right)$, with respect to $\mathcal{W}$, for the restriction $\sigma\left(\left[t, t^{\prime}\right)\right)$ of $\sigma$ to the interval $\left[t, t^{\prime}\right)$, where $|\sigma|$ is the duration of the signal. An illustration of $\mathcal{M}(\sigma, \mathcal{W})$ is in Fig. 1. In [BFMU17], quantitative timed pattern matching was solved by an offline algorithm using a syntax tree of signal regular expressions. In this paper, we propose an online algorithm for quantitative timed pattern matching with automata. To the best of our knowledge, this is the first online algorithm for quantitative timed pattern matching. Moreover, our (quantitative) semantics is parameterized by a semiring and what we call a cost function. This algebraic formulation makes our problem setting general.

Example 1. Let $\sigma$ be the piecewise-constant signal in the left of Fig. 1 and $\mathcal{W}$ be the specification meaning the following.

- At first, the value of $x$ stays less than 15 , and then the value of $x$ becomes and remains greater than 5 within $5 \mathrm{~s}$.

- We are only interested in the behavior within $10 \mathrm{~s}$ after the value of $x$ becomes greater than 5 .

- We want the score showing how robustly the above conditions are satisfied. 


$$
\begin{gathered}
\kappa_{r}\left(u,\left(a_{1} a_{2} \ldots a_{m}\right)\right)=\inf _{i \in\{1,2, \ldots, n\}} \kappa_{r}\left(u,\left(a_{i}\right)\right) \\
\kappa_{r}\left(\bigwedge_{i=1}^{n}\left(x_{i} \bowtie_{i} d_{i}\right),(a)\right)=\inf _{i \in\{1,2, \ldots, n\}} \kappa_{r}\left(x_{i} \bowtie_{i} d_{i},(a)\right) \text { where } \bowtie_{i} \in\{>, \geq, \leq,<\} \\
\kappa_{r}(x \succ d,(a))=a(x)-d \quad \text { where } \succ \in\{\geq,>\} \\
\kappa_{r}(x \prec d,(a))=d-a(x) \quad \text { where } \prec \in\{\leq,<\}
\end{gathered}
$$

Fig. 2: Example of a TSWA $\mathcal{W}=\left(\mathcal{A}, \kappa_{r}\right)$ which is the pair of the TSA $\mathcal{A}$ (upper) and the cost function $\kappa_{r}$ (lower). See Definition 5 for the precise definition.

The right of Fig. 1 illustrates the result of quantitative timed pattern matching. Quantitative timed pattern matching computes the semantics $(\mathcal{M}(\sigma, \mathcal{W}))\left(t, t^{\prime}\right)$, with respect to $\mathcal{W}$, for each sub-signal $\sigma\left(\left[t, t^{\prime}\right)\right)$ of $\sigma$. The current semantics shows how robustly the conditions are satisfied. The semantics $(\mathcal{M}(\sigma, \mathcal{W}))(3,15)$ for the sub-signal $\sigma([3,15))$ is 5 , which is the value at $(3,15)$ in the right of Fig. 1 . This is because the distance between the first constraint $x<15$ and the first valuation $x=10$ of the sub-signal $\sigma([3,15))$ is 5 , and the distance between the second constraint $x>5$ and the valuations $x=10, x=40$, and $x=60$ of the sub-signal $\sigma([3,15))$ is not smaller than 5 . The semantics $(\mathcal{M}(\sigma, \mathcal{W}))(10,15)$ for the sub-signal $\sigma([10,15))$ is -25 , which is the value at $(10,15)$ in the right of Fig. 1. Thus, the sub-signal $\sigma([3,15))$ satisfies the condition specified in $\mathcal{W}$ more robustly than the sub-signal $\sigma([10,15))$.

Our algorithm is online and it starts returning the result before obtaining the entire signal $\sigma$. For example, after obtaining the sub-signal $\sigma([0,7.5))$ of the initial $7.5 \mathrm{~s}$, our algorithm returns that for any $\left[t, t^{\prime}\right) \subseteq[0,7.5)$, the score $(\mathcal{M}(\sigma, \mathcal{W}))\left(t, t^{\prime}\right)$ is 5 .

Our solution We formulate quantitative timed pattern matching using the shortest distance [Moh09] of semiring-valued (potentially infinite) weighted graphs. We reduce it to the shortest distance of finite weighed graphs. This is in contrast with the qualitative setting: the semantics is defined by the reachability in a (potentially infinite) graph and it is reduced to the reachability in a finite graph. The following is an overview.

Problem formulation We introduce timed symbolic weighted automata (TSWAs) and define the (quantitative) semantics $\alpha(\sigma, \mathcal{W})$ of a signal $\sigma$ with respect to a TSWA $\mathcal{W}$. Moreover, we define quantitative timed pattern matching for a signal and a TSWA. A TSWA $\mathcal{W}$ is a pair $(\mathcal{A}, \kappa)$ of a timed symbolic automaton (TSA) $\mathcal{A}$ - that we also introduce in this paper - and a cost function $\kappa$. The cost function $\kappa$ returns a semiring value at each transition of $\mathcal{A}$, and the semiring operations specify how to accumulate such values over time. This algebraic definition makes our problem general. Fig. 2 shows an example of a TSWA. 


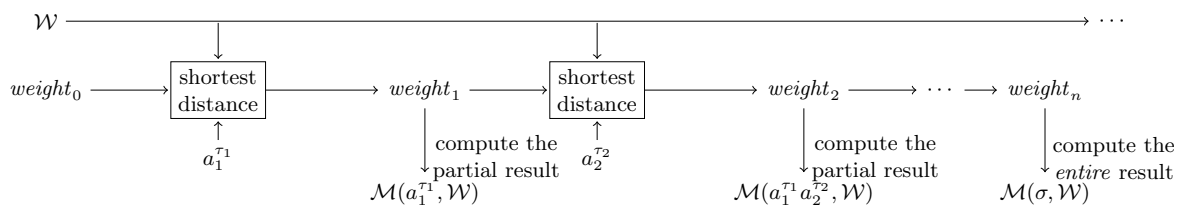

Fig. 3: Illustration of our online algorithm for quantitative timed pattern matching of a signal $\sigma=a_{1}^{\tau_{1}} a_{2}^{\tau_{2}} \cdots a_{n}^{\tau_{n}}$ meaning "the signal value is $a_{1}$ for $\tau_{1}$, the signal value is $a_{2}$ for the next $\tau_{2}, \ldots$ " and a TSWA $\mathcal{W}$. The intermediate data weight for the weight computation is represented by zones. The precise definition of the weight $_{i}$ is introduced later in Definition 16.

Algorithm by zones We give an algorithm for computing our semantics $\alpha(\sigma, \mathcal{W})$ of a signal $\sigma$ by the shortest distance of a finite weighted graph. The constructed weighted graph is much like the zone graph [BY03] for reachability analysis of timed automata. Our algorithm is general and works for any semantics defined on an idempotent and complete semiring. (See Example 4 later for examples of such semirings.)

Incremental and online algorithms We present an incremental algorithm for computing the semantics $\alpha(\sigma, \mathcal{W})$ of a signal $\sigma$ with respect to the TSWA $\mathcal{W}$. Based on this incremental algorithm for computing $\alpha(\sigma, \mathcal{W})$, we present an online algorithm for quantitative timed pattern matching. To the best of our knowledge, this is the first online algorithm for quantitative timed pattern matching. Our online algorithm for quantitative timed pattern matching works incrementally, much like in dynamic programming. Fig. 3 shows an illustration.

Contribution We summarize our contributions as follows.

- We formulate the semantics of a signal with respect to a TSWA by a shortest distance of a potentially infinite weighted graph.

- We reduce the above graph to a finite weighted graph.

- We give an online algorithm for quantitative timed pattern matching.

Related work Table 1 shows a comparison of the present study with some related studies. Since the formulation of qualitative timed pattern matching [UFAM14], many algorithms have been presented [UFAM14,UFAM16,WAH16,WHS17, $\mathrm{BFN}^{+}$18], including the online algorithms [WHS17, $\mathrm{BFN}^{+}$18] using timed automata. In the consequence, two tools have been presented [Ulu17,WHS18]. Quantitative timed pattern matching was formulated and solved by an offline algorithm in [BFMU17]. This offline algorithm is based on the syntax trees of signal regular expressions, and it is difficult to extend for online monitoring. Weighted automata are used for quantitative monitoring in [CHO16, $\left.\mathrm{JBG}^{+} 18, \mathrm{JBGN} 18\right]$, but the time model was discrete.

The online quantitative monitoring for signal temporal logic $\left[\mathrm{DDG}^{+} 15\right]$ is one of the closest work. Since we use the clock variables of TSAs to represent 
the intervals of timed pattern matching, it seems hard to use the algorithm in $\left[\mathrm{DDG}^{+} 15\right]$ for quantitative timed pattern matching.

Parametric timed pattern matching [AHW18,WA19] is another orthogonal extension of timed pattern matching, where timing constraints are parameterized. Symbolic monitoring [WAH] is a further generalization to handle infinite domain data i. e., real values and string labels. These problems answer feasible parameter valuations and different from our problem.

Organization of the paper Section 2 introduces preliminaries on signals and semirings. Section 3 defines timed symbolic weighted automata (TSWAs), and our quantitative semantics of signals over a TSWA. Section 4 defines the quantitative timed pattern matching problem. Section 5 and Section 6 describe our algorithms for computing the quantitative semantics and the quantitative timed pattern matching problem, respectively. Section 7 presents our experimental results for the sup-inf and tropical semirings, which confirm the scalability of our algorithm under some reasonable assumptions. Section 8 presents conclusions and some future perspectives.

\section{Preliminary}

For a set $X$, its powerset is denoted by $\mathcal{P}(X)$. We use $\varepsilon$ to represent the empty sequence. All the signals in this paper are piecewise-constant, which is one of the most common interpolation methods of sampled signals.

Definition 2 (signal). Let $X$ be a finite set of variables defined over a data domain $\mathbb{D}$. A (piecewise-constant) signal $\sigma$ is a sequence $\sigma=a_{1}^{\tau_{1}} a_{2}^{\tau_{2}} \cdots a_{n}^{\tau_{n}}$, where for each $i \in\{1,2, \ldots, n\}, a_{i} \in \mathbb{D}^{X}$ and $\tau_{i} \in \mathbb{R}_{>0}$. The set of signals over $\mathbb{D}^{X}$ is denoted by $\mathcal{T}\left(\mathbb{D}^{X}\right)$. The duration $\sum_{i=1}^{n} \tau_{i}$ of a signal $\sigma$ is denoted by $|\sigma|$. The sequence $a_{1} \circ a_{2} \circ \cdots \circ a_{n}$ of the values of a signal $\sigma$ is denoted by Values $(\sigma)$, where $a \circ a^{\prime}$ is the absorbing concatenation

$$
a \circ a^{\prime}=\left\{\begin{array}{ll}
a a^{\prime} & \text { if } a \neq a^{\prime} \\
a & \text { if } a=a^{\prime}
\end{array} .\right.
$$

We denote the set $\left\{a_{1} \circ a_{2} \circ \ldots \circ a_{n} \mid n \in \mathbb{Z}_{\geq 0}, a_{1}, a_{2}, \ldots, a_{n} \in \mathbb{D}^{X}\right\}$ by $\left(\mathbb{D}^{X}\right)^{\circledast}$. For $t \in[0,|\sigma|)$, we define $\sigma(t)=a_{k}$, where $k$ is such that $\sum_{i=1}^{k-1} \tau_{i} \leq t<\sum_{i=1}^{k} \tau_{i}$. For an interval $\left[t, t^{\prime}\right) \subseteq[0,|\sigma|)$, we define $\sigma\left(\left[t, t^{\prime}\right)\right)=$ $a_{k}^{\sum_{i=1}^{k} \tau_{i}-t} a_{k+1}^{\tau_{k+1}} \ldots a_{l-1}^{\tau_{l-1}} \ldots a_{l}^{t^{\prime}-\sum_{i=1}^{l-1} \tau_{i}}$, where $k$ and $l$ are such that $\sum_{i=1}^{k-1} \tau_{i} \leq$ $t<\sum_{i=1}^{k} \tau_{i}$ and $\sum_{i=1}^{l-1} \tau_{i}<t^{\prime} \leq \sum_{i=1}^{l} \tau_{i}$.

Definition 3 (semiring). A system $\mathbb{S}=\left(S, \oplus, \otimes, e_{\oplus}, e_{\otimes}\right)$ is a semiring if we have the following.

- $\left(S, \oplus, e_{\oplus}\right)$ is a commutative monoid with identity element $e_{\oplus}$.

- $\left(S, \otimes, e_{\otimes}\right)$ is a monoid with identity element $e_{\otimes}$. 
- For any $s, s^{\prime}, s^{\prime \prime} \in S$, we have $\left(s \oplus s^{\prime}\right) \otimes s^{\prime \prime}=\left(s \otimes s^{\prime \prime}\right) \oplus\left(s^{\prime} \otimes s^{\prime \prime}\right)$ and $s \otimes\left(s^{\prime} \oplus s^{\prime \prime}\right)=\left(s \otimes s^{\prime}\right) \oplus\left(s \otimes s^{\prime \prime}\right)$.

- For any $s \in S$, we have $e_{\oplus} \otimes s=s \otimes e_{\oplus}=e_{\oplus}$.

A semiring $\left(S, \oplus, \otimes, e_{\oplus}, e_{\otimes}\right)$ is complete if for any $S^{\prime} \subseteq S, \bigoplus_{s \in S^{\prime}} s$ is an element of $S$ satisfying the following.

$$
\begin{array}{rlrl}
\bigoplus_{s \in S^{\prime}} s & =e_{\oplus} \quad \text { if } S^{\prime}=\emptyset & \bigoplus_{s \in S^{\prime}} s=s \quad \text { if } S^{\prime}=\{s\} \\
\bigoplus_{s \in S^{\prime}} s & =\bigoplus_{i \in I}\left(\bigoplus_{s \in S_{i}^{\prime}} s\right) \quad \text { for any partition } S^{\prime}=\coprod_{i \in I} S_{i}^{\prime} & \\
s \otimes\left(\bigoplus_{s^{\prime} \in S^{\prime}} s^{\prime}\right) & =\bigoplus_{s^{\prime} \in S^{\prime}}\left(s \otimes s^{\prime}\right) \quad \text { and } \quad\left(\bigoplus_{s \in S^{\prime}} s\right) \otimes s^{\prime}=\bigoplus_{s \in S^{\prime}}\left(s \otimes s^{\prime}\right) \quad \text { for any } s \in S
\end{array}
$$

A semiring $\mathbb{S}=\left(S, \oplus, \otimes, e_{\oplus}, e_{\otimes}\right)$ is idempotent if for any $s \in S, s \oplus s=s$ holds. For a semiring $\left(S, \oplus, \otimes, e_{\oplus}, e_{\otimes}\right)$ and $s_{1}, s_{2}, \ldots, s_{n} \in S$, we denote $\bigoplus_{i=1}^{n} s_{i}=$ $s_{1} \oplus s_{2} \oplus \cdots \oplus s_{n}$ and $\bigotimes_{i=1}^{n} s_{i}=s_{1} \otimes s_{2} \otimes \cdots \otimes s_{n}$.

Example 4. The Boolean semiring $(\{\top, \perp\}, \vee, \wedge, \perp, \top)$, the sup-inf semiring ( $\mathbb{R} \amalg$ $\{ \pm \infty\}$, sup, inf, $-\infty,+\infty)$, and the tropical semiring ( $\mathbb{R} \amalg\{+\infty\}$, inf, $+,+\infty, 0)$ are complete and idempotent.

Let $\mathbb{S}=\left(S, \oplus, \otimes, e_{\oplus}, e_{\otimes}\right)$ be a semiring and $G=(V, E, W)$ be a weighted graph over $\mathbb{S}$, i.e., $V$ is the finite set of vertices, $E \subseteq V \times V$ is the finite set of edges, and $W: V \times V \rightarrow \mathbb{S}$ is the weight function. For $V_{\text {from }}, V_{\text {to }} \subseteq$ $V$, the shortest distance from $V_{\text {from }}$ to $V_{\text {to }}$ is $\operatorname{Dist}\left(V_{\text {from }}, V_{\text {to }}, V, E, W\right)=$ $\bigoplus_{v \in V_{\text {from }, v^{\prime} \in V_{\text {to }}}} \bigoplus_{v=v_{1} v_{2} \ldots v_{n}=v^{\prime} \in \operatorname{Paths}(G)} \bigotimes_{i=1}^{n-1} W\left(v_{i}, v_{i+1}\right)$, where Paths $(G)$ is the set of the paths in the directed graph $G$, i.e., Paths $(G)=\left\{v_{1} v_{2} \ldots v_{n} \mid\right.$ $\left.\forall i \in\{1,2, \ldots, n-1\} .,\left(v_{i}, v_{i+1}\right) \in E\right\}$. For any complete semiring, the shortest distance problem can be solved by a generalization of the Floyd-Warshall algorithm [Moh09]. Under some conditions, the shortest distance problem can be solved more efficiently by a generalization of the Bellman-Ford algorithm [Moh09].

\section{Timed symbolic weighted automata}

We propose timed symbolic automata (TSAs), timed symbolic weighted automata (TSWAs), and the (quantitative) semantics of TSWAs. TSAs are an adaptation of timed automata [AD94] for handling signals over $\mathbb{D}$ rather than signals over a finite alphabet. In the remainder of this paper, we assume that the data domain $\mathbb{D}$ is equipped with a partial order $\leq$. A typical example of the data domain $\mathbb{D}$ is the reals $\mathbb{R}$ with the usual order. We note that TSAs are much like the state-based variant of timed automata $\left[\mathrm{ACM} 97, \mathrm{BFN}^{+} 18\right]$ rather than the original, event-based definition [AD94].

For a finite set $X$ of variables and a poset $(\mathbb{D}, \leq)$, we denote by $\Phi(X, \mathbb{D})$ the set of constraints defined by a finite conjunction of inequalities $x \bowtie d$, where 
$x \in X, d \in \mathbb{D}$, and $\bowtie \in\{>, \geq,<, \leq\}$. We denote $\bigwedge \emptyset \in \Phi(X, \mathbb{D})$ by $\top$. For a finite set $C$ of clock variables, a clock valuation is a function $\nu \in\left(\mathbb{R}_{\geq 0}\right)^{C}$. For a clock valuation $\nu \in\left(\mathbb{R}_{\geq 0}\right)^{C}$ over $C$ and $C^{\prime} \subseteq C$, we let $\nu \downarrow_{C^{\prime}} \in\left(\mathbb{R}_{\geq 0}\right)^{C^{\prime}}$ be the clock valuation over $C^{\prime}$ satisfying $\nu \downarrow_{C^{\prime}}(c)=\nu(c)$ for any $c \in C^{\prime}$. For a finite set $C$ of clock variables, let $\mathbf{0}_{C}$ be the clock valuation $\mathbf{0}_{C} \in\left(\mathbb{R}_{>0}\right)^{C}$ satisfying $\mathbf{0}_{C}(c)=0$ for any $c \in C$. For a clock valuation $\nu$ over $C$ and $\tau \in \mathbb{R}_{\geq 0}$, we denote by $\nu+\tau$ the valuation satisfying $(\nu+\tau)(c)=\nu(c)+\tau$ for any $c \in \bar{C}$. For a clock valuation $\nu \in\left(\mathbb{R}_{\geq 0}\right)^{C}$ and $\rho \subseteq C$, we denote by $\nu[\rho:=0]$ the valuation such that $(\nu[\rho:=0])(x)=0$ for $c \in \rho$ and $(\nu[\rho:=0](c)=\nu(c)$ for $c \notin \rho$.

The definitions of TSAs and TSWAs are as follows. As shown in Fig. 2, TSAs are similar to the timed automata in $\left[\mathrm{ACM} 97, \mathrm{BFN}^{+} 18\right]$, but the locations are labeled with a constraint on the signal values $\mathbb{D}^{X}$ instead of a character in a finite alphabet.

Definition 5 (timed symbolic, timed symbolic weighted automata). For a poset $(\mathbb{D}, \leq)$, a timed symbolic automaton (TSA) over $\mathbb{D}$ is a 7-tuple $\mathcal{A}=\left(X, L, L_{0}, L_{F}, C, \Delta, \Lambda\right)$, where:

- $X$ is a finite set of variables over $\mathbb{D}$;

- $L$ is the finite set of locations;

- $L_{0} \subseteq L$ is the set of initial locations;

- $L_{F} \subseteq L$ is the set of accepting locations;

- $C$ is the finite set of clock variables;

- $\Delta \subseteq L \times \Phi\left(C, \mathbb{Z}_{\geq 0}\right) \times \mathcal{P}(C) \times L$ is the set of transitions; and

- $\Lambda$ is the labeling function $\Lambda: L \rightarrow \Phi(X, \mathbb{D})$.

For a poset $(\mathbb{D}, \leq)$ and a complete semiring $\mathbb{S}=\left(S, \oplus, \otimes, e_{\oplus}, e_{\otimes}\right)$, a timed symbolic weighted automaton $(T S W A)$ over $\mathbb{D}$ and $\mathbb{S}$ is a pair $\mathcal{W}=(\mathcal{A}, \kappa)$ of a $T S A \mathcal{A}$ over $\mathbb{D}$ and a cost function $\kappa: \Phi(X, \mathbb{D}) \times\left(\mathbb{D}^{X}\right)^{\circledast} \rightarrow S$ over $\mathbb{S}$.

The semantics of a TSWA $\mathcal{W}=(\mathcal{A}, \kappa)$ on a signal $\sigma$ is defined by the trace value $\alpha(\mathcal{S})$ of the weighted timed transition systems (WTTS) $\mathcal{S}$ of $\sigma$ and $\mathcal{W}$. The trace value $\alpha(\mathcal{S})$ depends on the cost function $\kappa$ and implicitly on its range semiring $\mathbb{S}$ as well as the signal $\sigma$ and the TSA $\mathcal{A}$. As shown below, the state space of a WTTS $\mathcal{S}$ is $Q=L \times\left(\mathbb{R}_{\geq 0}\right)^{C} \times[0,|\sigma|] \times\left(\mathbb{D}^{X}\right)^{\circledast}$. Intuitively, a state $(l, \nu, t, \bar{a}) \in Q$ of $\mathcal{S}$ consists of: the current location $l$; the current clock valuation $\nu$; the current absolute time $t$; and the observed signal value $\bar{a}$ after the latest transition. The transition $\rightarrow$ of $\mathcal{S}$ is for a transition of $\mathcal{A}$ or time elapse.

Definition 6 (weighted timed transition systems). For a signal $\sigma \in$ $\mathcal{T}\left(\mathbb{D}^{X}\right)$ and a TSWA $\mathcal{W}=(\mathcal{A}, \kappa)$ over the data domain $\mathbb{D}$ and semiring $\mathbb{S}$, the weighted timed transition system (WTTS) $\mathcal{S}=\left(Q, Q_{0}, Q_{F}, \rightarrow, W\right)$ is as follows, where $\mathcal{A}=\left(X, L, L_{0}, L_{F}, C, \Delta, \Lambda\right)$ is a TSA over $\mathbb{D}$ and $\kappa$ is a cost function over $\mathbb{S}$.

- $Q=L \times\left(\mathbb{R}_{\geq 0}\right)^{C} \times[0,|\sigma|] \times\left(\mathbb{D}^{X}\right)^{\circledast}$

- $Q_{0}=\left\{\left(l_{0}, \mathbf{0}_{C}, 0, \varepsilon\right) \mid l_{0} \in L_{0}\right\}$

- $Q_{F}=\left\{\left(l_{F}, \nu,|\sigma|, \varepsilon\right) \mid l_{F} \in L_{F}, \nu \in\left(\mathbb{R}_{\geq 0}\right)^{C}\right\}$ 
- $\rightarrow \subseteq Q \times Q$ is the relation such that $\left((l, \nu, t, \bar{a}),\left(l^{\prime}, \nu^{\prime}, t^{\prime}, \overline{a^{\prime}}\right)\right) \in \rightarrow$ if and only if either of the following holds.

(transition of $\mathcal{A}) \exists\left(l, g, \rho, l^{\prime}\right) \in \Delta$ satisfying $\nu \mid=g, \nu^{\prime}=\nu[\rho:=0], t^{\prime}=t$, $\overline{a^{\prime}}=\varepsilon$, and $\bar{a} \neq \varepsilon$

(time elapse) $\exists \tau \in \mathbb{R}_{>0}$ satisfying $l=l^{\prime}, \nu^{\prime}=\nu+\tau, t^{\prime}=t+\tau$, and $\overline{a^{\prime}}=\bar{a} \circ \operatorname{Values}(\sigma([t, t+\tau)))$

- $W\left((l, \nu, t, \bar{a}),\left(l^{\prime}, \nu^{\prime}, t^{\prime}, \overline{a^{\prime}}\right)\right)$ is $\kappa(\Lambda(l), \bar{a})$ if $\overline{a^{\prime}}=\varepsilon$; and $e_{\otimes}$ if $\overline{a^{\prime}} \neq \varepsilon$

Definition 7 (trace value). For a WTTS $\mathcal{S}=\left(Q, Q_{0}, Q_{F}, \rightarrow, W\right)$, the trace value $\alpha(\mathcal{S})$ is the shortest distance $\operatorname{Dist}\left(Q_{0}, Q_{F}, Q, \rightarrow, W\right)$ from $Q_{0}$ to $Q_{F}$.

For a signal $\sigma$ and a TSWA $\mathcal{W}$, by $\alpha(\sigma, \mathcal{W})$, we denote the trace value $\alpha(\mathcal{S})$ of the WTTS $\mathcal{S}$ of $\sigma$ and $\mathcal{W}$.

Example 8. By changing the semiring $\mathbb{S}$ and the cost function $\kappa$, various semantics can be defined by the trace value. Let $\mathbb{D}=\mathbb{R}$. For the Boolean semiring $(\{\top, \perp\}, \vee, \wedge, \perp, \top)$ in Example 4, the following function $\kappa_{b}$ is a prototypical example of a cost function, where $u \in \Phi(X, \mathbb{D})$ and $\left(a_{1} a_{2} \ldots a_{m}\right) \in\left(\mathbb{D}^{X}\right)^{\circledast}$.

$$
\begin{aligned}
\kappa_{b}\left(u,\left(a_{1} a_{2} \ldots a_{m}\right)\right) & =\bigwedge_{i=1}^{m} \kappa_{b}\left(u,\left(a_{i}\right)\right) \\
\kappa_{b}\left(\bigwedge_{i=1}^{n}\left(x_{i} \bowtie_{i} d_{i}\right),(a)\right) & =\bigwedge_{i=1}^{n} \kappa_{b}\left(x_{i} \bowtie_{i} d_{i},(a)\right) \quad \text { where } \bowtie_{i} \in\{>, \geq, \leq,<\} \\
\kappa_{b}(x \bowtie d,(a)) & = \begin{cases}\top & \text { if } a \mid=x \bowtie d \\
\perp & \text { if } a \mid f x \bowtie d\end{cases}
\end{aligned}
$$

For the sup-inf semiring ( $\mathbb{R} \amalg\{ \pm \infty\}$, sup, inf, $-\infty,+\infty)$ in Example 4 , the trace value defined by the cost function $\kappa_{r}$ in Fig. 2 captures the essence of the so-called space robustness [FP09,BFMU17]. For the tropical semiring $(\mathbb{R} \amalg\{+\infty\}$, inf, $+,+\infty, 0)$ in Example 4, an example cost function $\kappa_{t}$ is as follows.

$$
\begin{aligned}
\kappa_{t}\left(u,\left(a_{1} a_{2} \ldots a_{m}\right)\right) & =\sum_{i=1}^{n} \kappa_{r}\left(u,\left(a_{i}\right)\right) \\
\kappa_{t}\left(\bigwedge_{i=1}^{n}\left(x_{i} \bowtie_{i} d_{i}\right),(a)\right) & =\sum_{i=1}^{n} \kappa_{t}\left(x_{i} \bowtie_{i} d_{i},(a)\right) \quad \text { where } \bowtie_{i} \in\{>, \geq, \leq,<\} \\
\kappa_{t}(x \succ d,(a)) & =a(x)-d \quad \text { where } \succ \in\{\geq,>\} \\
\kappa_{t}(x \prec d,(a)) & =d-a(x) \quad \text { where } \prec \in\{\leq,<\}
\end{aligned}
$$

Example 9. Let $\mathcal{W}=(\mathcal{A}, \kappa)$ be a TSWA over $\mathbb{R}$ and $\mathbb{S}$, where $\mathcal{A}$ is the TSA over $\mathbb{R}$ in Fig. 2, $\sigma$ be the signal $\sigma=\{x=10\}^{2.5}\{x=40\}^{1.0}\{x=60\}^{3.0}$. When $\mathbb{S}=(\mathbb{R} \amalg\{ \pm \infty\}$, sup, inf, $-\infty,+\infty)$ and $\kappa$ is the cost function $\kappa_{r}$ in Example 8 , we have $\alpha(\sigma, \mathcal{W})=5$. When $\mathbb{S}=(\mathbb{R} \amalg\{+\infty\}$, inf, $+,+\infty, 0)$ and $\kappa$ is the cost function $\kappa_{t}$ in Example 8, we have $\alpha(\sigma, \mathcal{W})=35$. 


\section{Quantitative timed pattern matching}

Using TSWAs, we formulate quantitative timed pattern matching as follows.

Definition 10 (quantitative timed pattern matching). For a TSWA $\mathcal{W}$ over the data domain $\mathbb{D}$ and complete semiring $\mathbb{S}$, and a signal $\sigma \in \mathcal{T}\left(\mathbb{D}^{X}\right)$, the quantitative matching function $\mathcal{M}(\sigma, \mathcal{W}): \operatorname{dom}(\sigma) \rightarrow S$ is $(\mathcal{M}(\sigma, \mathcal{W}))\left(t, t^{\prime}\right)=$ $\alpha(\sigma([t, t)), \mathcal{W})$, where $\operatorname{dom}(\sigma)=\left\{\left(t, t^{\prime}\right)\left|0 \leq t<t^{\prime} \leq\right| \sigma \mid\right\}$ and $S$ is the underlying set of $\mathbb{S}$. Given a signal $\sigma \in \mathcal{T}\left(\mathbb{D}^{X}\right)$ and a TSWA $\mathcal{W}$ over the data domain $\mathbb{D}$ and complete semiring $\mathbb{S}$, the quantitative timed pattern matching problem asks for the quantitative matching function $\mathcal{M}(\sigma, \mathcal{W})$.

Example 11. Let $\mathcal{W}$ be the TSWA shown in Fig. 2, which is defined over the reals $\mathbb{R}$ and the sup-inf semiring $(\mathbb{R} \amalg\{ \pm \infty\}$, sup, inf, $-\infty,+\infty)$, and $\sigma$ be the signal $\sigma=\{x=10\}^{7.5}\{x=40\}^{10.0}\{x=60\}^{13.0}$. The quantitative matching function $\mathcal{M}(\sigma, \mathcal{W})$ is as follows. Fig. 1 shows an illustration.

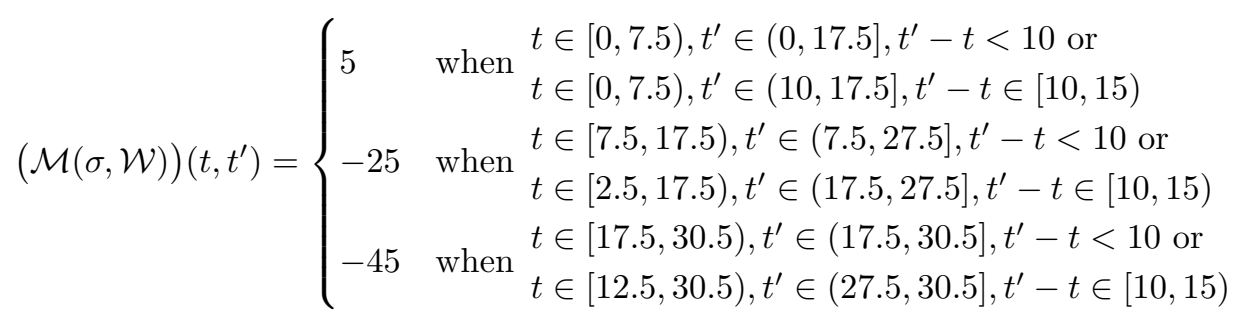

Although the domain $\left.\left\{\left(t, t^{\prime}\right)\left|0 \leq t<t^{\prime} \leq\right| \sigma \mid\right)\right\}$ of the quantitative matching function $\mathcal{M}(\sigma, \mathcal{W})$ is an infinite set, $\mathcal{M}(\sigma, \mathcal{W})$ is a piecewise-constant function with finitely many pieces. Moreover, each piece of $\mathcal{M}(\sigma, \mathcal{W})$ can be represented by a special form of convex polyhedra called zones [Dil89].

Definition 12 (zone). For a finite set of clock variables $C$, a zone is a $|C|$ dimensional convex polyhedron defined by a finite conjunction of the constraints of the form $c \bowtie d$ or $c-c^{\prime} \bowtie d$, where $c, c^{\prime} \in C, \bowtie \in\{>, \geq, \leq,<\}$, and $d \in \mathbb{R}$. The set of zones over $C$ is denoted by $\mathcal{Z}(C)$. By a zone $Z \in \mathcal{Z}(C)$, we also represent the set $\{\nu \mid \nu \models Z\} \subseteq\left(\mathbb{R}_{\geq 0}\right)^{C}$ of clock valuations.

Theorem 13. For any TSWA $\mathcal{W}$ over $\mathbb{D}$ and $\mathbb{S}$ and for any signal $\sigma \in \mathcal{T}\left(\mathbb{D}^{X}\right)$, there is a finite set $\left\{\left(Z_{1}, s_{1}\right),\left(Z_{2}, s_{2}\right), \ldots,\left(Z_{n}, s_{n}\right)\right\} \subseteq \mathcal{Z}\left(\left\{c_{\text {begin }}, c_{\text {end }}\right\}\right) \times S$ such that $Z_{1}, Z_{2}, \ldots, Z_{n}$ is a partition of the domain $\left\{\left(t, t^{\prime}\right)\left|0 \leq t<t^{\prime} \leq\right| \sigma \mid\right\}$, and for any $\left[t, t^{\prime}\right) \subseteq \mathbb{R}_{\geq 0}$ satisfying $0 \leq t<t^{\prime} \leq|\sigma|$, there exists $i \in\{1,2, \ldots, n\}$ and $\nu \in Z_{i}$ satisfying $\nu\left(c_{\text {begin }}\right)=t, \nu\left(c_{\text {end }}\right)=t^{\prime}$, and $(\mathcal{M}(\sigma, \mathcal{W}))\left(t, t^{\prime}\right)=s_{i}$.

\section{Trace value computation by shortest distance}

We present an algorithm to compute the trace values $\alpha(\mathcal{S})$. Since a WTTS possibly has infinitely many states and transitions (see Definition 6), we need a finite abstraction of it. We use zone-based abstraction for what we call weighted symbolic timed transition systems (WSTTSs). In addition to the clock variables in the TSA, we introduce a fresh clock variable $T$ to represent the absolute time. 


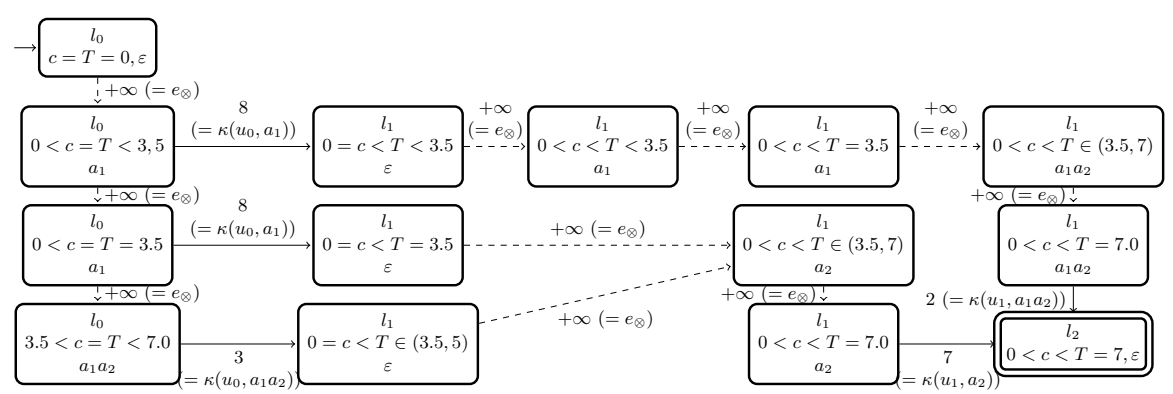

Fig. 4: WSTTS $\mathcal{S}^{\mathrm{sym}}$ of the TSWA $\mathcal{W}$ in Fig. 2 and the signal $\sigma=a_{1}^{3.5} a_{2}^{3.5}$, where $u_{0}=x<15, u_{1}=x>5, a_{1}=\{x=7\}$, and $a_{2}=\{x=12\}$. The states unreachable from the initial state or unreachable to the accepting state are omitted. The transition for time elapse which can be represented by the composition of other transitions are also omitted. A dashed transition is for the time elapse and a solid transition is for a transition of $\mathcal{A}$.

Definition 14 (weighted symbolic timed transition system). For a TSWA $\mathcal{W}=(\mathcal{A}, \kappa)$ over the data domain $\mathbb{D}$ and complete semiring $\mathbb{S}$, and a signal $\sigma=a_{1}^{\tau_{1}} a_{2}^{\tau_{2}} \cdots a_{n}^{\tau_{n}} \in \mathcal{T}\left(\mathbb{D}^{X}\right)$, where $\mathcal{A}=\left(X, L, L_{0}, L_{F}, C, \Delta, \Lambda\right)$, the weighted symbolic timed transition system (WSTTS) is the 5-tuple $\mathcal{S}^{\mathrm{sym}}=$ $\left(Q^{\mathrm{sym}}, Q_{0}^{\mathrm{sym}}, Q_{F}^{\mathrm{sym}}, \rightarrow^{\mathrm{sym}}, W^{\mathrm{sym}}\right)$ defined as follows.

- $Q^{\mathrm{sym}}=\left\{(l, Z, \bar{a}) \in L \times \mathcal{Z}(C \amalg\{T\}) \times\left(\mathbb{D}^{X}\right)^{\circledast}|Z \neq \emptyset, \forall \nu \in Z . \nu(T) \leq| \sigma \mid, \bar{a}=\right.$ $\varepsilon$ or $\bar{a} \circ \sigma(\nu(T))=\bar{a}\}$

- $Q_{0}^{\mathrm{sym}}=\left\{\left(l_{0},\left\{\mathbf{0}_{C \amalg\{T\}}\right\}, \varepsilon\right) \mid l_{0} \in L_{0}\right\}$

- $Q_{F}^{\mathrm{sym}}=\left\{\left(l_{F}, Z, \varepsilon\right)\left|l_{F} \in L_{F}, \exists \nu \in Z . \nu(T)=\right| \sigma \mid\right\}$

- $\rightarrow^{\mathrm{sym}} \subseteq Q^{\mathrm{sym}} \times Q^{\mathrm{sym}}$ is the relation such that $\left((l, Z, \bar{a}),\left(l^{\prime}, Z^{\prime}, \overline{a^{\prime}}\right)\right) \in \rightarrow^{\mathrm{sym}}$ if and only if one of the following holds.

(transition of $\mathcal{A})$ there exists $\left(l, g, \rho, l^{\prime}\right) \in \Delta$, satisfying $Z^{\prime}=\{\nu[\rho:=0] \mid$ $\nu \in Z, \nu \models g\}, \bar{a} \neq \varepsilon$, and $\overline{a^{\prime}}=\varepsilon$.

(punctual time elapse) $l=l^{\prime}, \overline{a^{\prime}}=\bar{a} \circ \operatorname{Values}\left(\sigma\left(\left[\tilde{\nu}(T), \tilde{\nu}^{\prime}(T)\right)\right)\right)$, and there is $i \in\{1,2, \ldots, n\}$ satisfying $Z^{\prime}=\left\{\nu+\tau \mid \nu \in Z, \tau \in \mathbb{R}_{>0}\right\} \cap M_{i,=}$, where $\tilde{\nu} \in Z, \tilde{\nu}^{\prime} \in Z^{\prime 1}, M_{i,=}=\left\{\nu \mid \nu(T)=\sum_{j=0}^{i} \tau_{j}\right\}$.

(non-punctual time elapse) $l=l^{\prime}, \overline{a^{\prime}}=\bar{a} \circ \operatorname{Values}\left(\sigma\left(\left[\tilde{\nu}(T), \tilde{\nu}^{\prime}(T)\right)\right)\right)$, and there is $i \in\{1,2, \ldots, n\}$ satisfying $Z^{\prime}=\left\{\nu+\tau \mid \nu \in Z, \tau \in \mathbb{R}_{>0}\right\} \cap M_{i}$, where $\tilde{\nu} \in Z, \tilde{\nu}^{\prime} \in Z^{\prime}$, and $M_{i}=\left\{\nu \mid \sum_{j=0}^{i-1} \tau_{j}<\nu(T)<\sum_{j=0}^{i} \tau_{j}\right\}$.

- $W^{\operatorname{sym}}\left((l, Z, \bar{a}),\left(l^{\prime}, Z^{\prime}, \overline{a^{\prime}}\right)\right)$ is $\kappa(\Lambda(l), \bar{a})$ if $\overline{a^{\prime}}=\varepsilon$; and $e_{\otimes}$ if $\overline{a^{\prime}} \neq \varepsilon$

Although the state space $Q^{\text {sym }}$ of the WSTTS $\mathcal{S}^{\text {sym }}$ may be infinite, there are only finitely many states reachable from $Q_{0}^{\text {sym }}$ and therefore, we can construct the reachable part of $\mathcal{S}^{\mathrm{sym}}$. See Appendix A.1 for the proof. An example of a WSTTS is shown in Fig. 4. For a WSTTS $\mathcal{S}^{\text {sym }}$, we define the symbolic trace value $\alpha^{\mathrm{sym}}\left(\mathcal{S}^{\mathrm{sym}}\right)$ as the shortest distance $\operatorname{Dist}\left(Q_{0}^{\mathrm{sym}}, Q_{F}^{\mathrm{sym}}, Q^{\mathrm{sym}}, \rightarrow^{\mathrm{sym}}, W^{\mathrm{sym}}\right)$ from $Q_{0}^{\text {sym }}$ to $Q_{F}^{\text {sym }}$.

\footnotetext{
${ }^{1}$ The choice of $\tilde{\nu}$ and $\tilde{\nu}^{\prime}$ does not change $\sigma(\tilde{\nu}(T))$ and $\sigma\left(\tilde{\nu}^{\prime}(T)\right)$ due to the definition of $Q^{\text {sym }}$.
} 


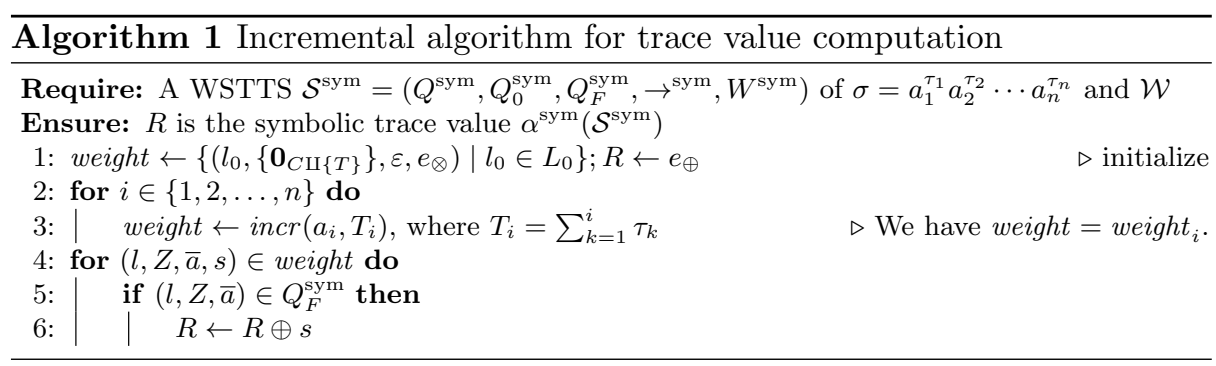

Theorem 15. Let $\mathcal{W}$ be a TSWA over $\mathbb{D}$ and $\mathbb{S}$, and $\sigma \in \mathcal{T}\left(\mathbb{D}^{X}\right)$ be a signal. Let $\mathcal{S}$ and $\mathcal{S}^{\mathrm{sym}}$ be the WTTS (in Definition 6) and WSTTS of $\mathcal{W}$ and $\sigma$, respectively. If $\mathbb{S}$ is idempotent, we have $\alpha(\mathcal{S})=\alpha^{\mathrm{sym}}\left(\mathcal{S}^{\mathrm{sym}}\right)$.

Because of Theorem 15, we can compute the trace value $\alpha(\mathcal{S})$ by

1. constructing the reachable part of $\mathcal{S}^{\mathrm{sym}}$; and

2. computing the symbolic trace value $\alpha^{\mathrm{sym}}\left(\mathcal{S}^{\mathrm{sym}}\right)$ using an algorithm for the shortest distance problem.

For example, the symbolic trace value of the WSTTS in Fig. 4 is $\alpha^{\mathrm{sym}}\left(\mathcal{S}^{\mathrm{sym}}\right)=$ $\max \{\min \{8,2\}, \min \{8,7\}, \min \{3,7\}\}=7$. However, this method requires the whole signal to compute the trace value, and it does not suit for the use in online quantitative timed pattern matching. Instead, we define the intermediate weight weight $_{i}$ and give an incremental algorithm to compute the trace value $\alpha(\mathcal{S})$. Intuitively, for each state $(l, Z, \bar{a}) \in Q^{\mathrm{sym}}$ of the WSTTS $\mathcal{S}^{\mathrm{sym}}$, the intermediate weight weight ${ }_{i}$ assign the shortest distance to reach $(l, Z, \bar{a})$ by reading the subsignal $a_{1}^{\tau_{1}} a_{2}^{\tau_{2}} \cdots a_{i}^{\tau_{i}}$ of $\sigma=a_{1}^{\tau_{1}} a_{2}^{\tau_{2}} \cdots a_{n}^{\tau_{n}}$.

Definition 16 (incr, weight in $_{i}$. For a TSWA $\mathcal{W}=(\mathcal{A}, \kappa)$ over the data domain $\mathbb{D}$ and complete semiring $\mathbb{S}, a \in \mathbb{D}^{X}$, and $t \in \mathbb{R}_{>0}$, the increment function

$$
\begin{aligned}
& \operatorname{incr}(a, t): \mathcal{P}\left(L \times \mathcal{Z}(C \amalg\{T\}) \times\left(\mathbb{D}^{X}\right)^{\circledast} \times S\right) \rightarrow \mathcal{P}\left(L \times \mathcal{Z}(C \amalg\{T\}) \times\left(\mathbb{D}^{X}\right)^{\circledast} \times S\right) \\
& \text { is as follows, where } \mathcal{A}=\left(X, L, L_{0}, L_{F}, C, \Delta, \Lambda\right) \text { and } \\
& \operatorname{incr}(a, t)(w)=\left\{\left(l^{\prime}, Z^{\prime}, \overline{a^{\prime}}, s^{\prime}\right) \in L \times \mathcal{Z}(C \amalg\{T\}) \times\left(\mathbb{D}^{X}\right)^{\circledast} \times S \mid \forall \nu^{\prime} \in Z^{\prime} . \nu^{\prime}(T)=t,\right. \\
& \left.s^{\prime}=\bigoplus_{(l, Z, \bar{a}, s) \in w} s \otimes \operatorname{Dist}\left(\{(l, Z, \bar{a})\},\left\{\left(l^{\prime}, Z^{\prime}, \overline{a^{\prime}}\right)\right\}, Q_{a, t}^{\mathrm{sym}}, \rightarrow_{a, t}^{\mathrm{sym}}, W_{a, t}^{\mathrm{sym}}\right)\right\}
\end{aligned}
$$

For a TSWA $\mathcal{W}$ over $\mathbb{D}$ and $\mathbb{S}$, a signal $\sigma=a_{1}^{\tau_{1}} a_{2}^{\tau_{2}} \cdots a_{n}^{\tau_{n}}$, and $i \in\{1,2, \ldots, n\}$, the intermediate weight weight ${ }_{i}$ is defined as follows, where $T_{j}=\sum_{k=1}^{j} \tau_{k}$.

$$
\text { weight }_{i}=\left(\operatorname{incr}\left(a_{i}, T_{i}\right) \circ \cdots \circ \operatorname{incr}\left(a_{1}, T_{1}\right)\right)\left(\left\{\left(l_{0},\left\{\mathbf{0}_{C \amalg\{T\}}\right\}, \varepsilon, e_{\otimes}\right) \mid l_{0} \in L_{0}\right\}\right)
$$

Because of the following theorem, we can incrementally compute the symbolic trace value $\alpha^{\mathrm{sym}}\left(\mathcal{S}^{\mathrm{sym}}\right)$, which is equal to the trace value $\alpha(\sigma, \mathcal{W})$, by Algorithm 1. 


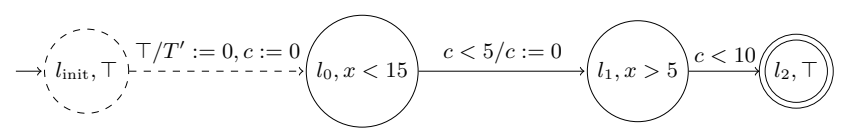

Fig. 5: Matching automaton $\mathcal{A}_{\text {match }}$ for the TSA $\mathcal{A}$ shown in Fig. 2. The fresh initial location $l_{\text {init }}$ and the transition to the original initial location $l_{0}$ are added.

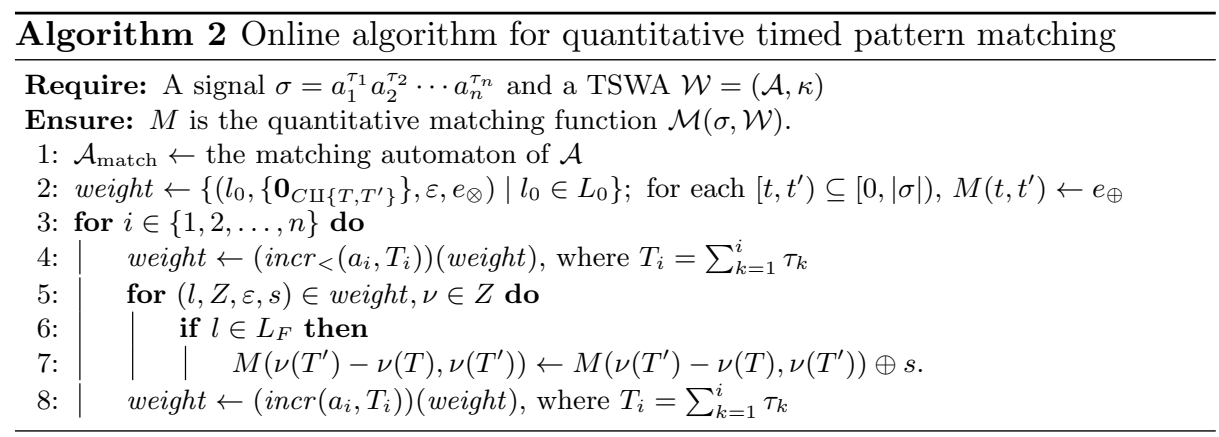

Theorem 17. For any WSTTS $\mathcal{S}^{\mathrm{sym}}$ of a signal $\sigma=a_{1}^{\tau_{1}} a_{2}^{\tau_{2}} \cdots a_{n}^{\tau_{n}}$ and a TSWA $\mathcal{W}$ over $\mathbb{D}$ and $\mathbb{S}$, we have the following, where $Q_{F}^{\mathrm{sym}}$ is the accepting states of $\mathcal{S}^{\text {sym }}$

$$
\alpha^{\mathrm{sym}}\left(\mathcal{S}^{\mathrm{sym}}\right)=\bigoplus_{(l, Z, \bar{a}) \in Q_{F}^{\mathrm{sym}}} \bigoplus_{(l, Z, \bar{a}, s) \in \text { weight }_{n}} s
$$

\section{Online algorithm for quantitative timed pattern matching}

In quantitative timed pattern matching, we compute the trace value $\alpha\left(\sigma\left(\left[t, t^{\prime}\right)\right), \mathcal{W}\right)$ for each sub-signal $\sigma\left(\left[t, t^{\prime}\right)\right)$. In order to try matching for each sub-signal $\sigma\left(\left[t, t^{\prime}\right)\right)$, we construct the matching automaton $\left[\mathrm{BFN}^{+} 18\right] \mathcal{A}_{\text {match }}$ from the TSA $\mathcal{A}$. The matching automaton $\mathcal{A}_{\text {match }}$ is constructed by adding a new clock variable $T^{\prime}$ and a new initial state $l_{\text {init }}$ to the TSA $\mathcal{A}$. The new clock variable $T^{\prime}$ represents the duration from the beginning $t$ of the sub-signal $\sigma\left(\left[t, t^{\prime}\right)\right)$. The new state $l_{\text {init }}$ is used to start the sub-signal in the middle of the signal. We add transitions from $l_{\text {init }}$ to each initial state $l_{0}$ of $\mathcal{A}$, resetting all of the clock variables. Fig. 5 shows an example of $\mathcal{A}_{\text {match }}$. We also define the auxiliary $i n c r<$ for our online algorithm for quantitative timed pattern matching.

Definition 18 (matching automaton $\left[\mathrm{BFN}^{+}\right.$18] $\mathcal{A}_{\text {match }}$ ). For a $T S A \mathcal{A}=$ $\left(X, L, L_{0}, L_{F}, C, \Delta, \Lambda\right)$ over $\mathbb{D}$, the matching automaton is the TSA $\mathcal{A}_{\text {match }}=$ $\left(X, L \amalg\left\{l_{\text {init }}\right\},\left\{l_{\text {init }}\right\}, L_{F}, C \amalg\left\{T^{\prime}\right\}, \Delta^{\prime}, \Lambda^{\prime}\right)$ over $\mathbb{D}$, where the transition is $\Delta^{\prime}=$ $\Delta \amalg\left\{\left(l_{\text {init }}, \top, C \amalg\left\{T^{\prime}\right\}, l_{0}\right) \mid l_{0} \in L_{0}\right\}$, the labeling function is $\Lambda^{\prime}\left(l_{\text {init }}\right)=\top$, and $\Lambda^{\prime}(l)=\Lambda(l)$ for $l \in L$. 
Definition $19\left(\right.$ incr $\left._{<}\right)$. For a TSWA $\mathcal{W}=(\mathcal{A}, \kappa)$ over the data domain $\mathbb{D}$ and complete semiring $\mathbb{S}, a \in \mathbb{D}^{X}$, and $t \in \mathbb{R}_{>0}$, the partial increment function

$$
\begin{aligned}
& \operatorname{incr}_{<}(a, t): \mathcal{P}\left(L \times \mathcal{Z}(C \amalg\{T\}) \times\left(\mathbb{D}^{X}\right)^{\circledast} \times S\right) \rightarrow \mathcal{P}\left(L \times \mathcal{Z}(C \amalg\{T\}) \times\left(\mathbb{D}^{X}\right)^{\circledast} \times S\right) \\
& \text { is as follows, where } \mathcal{A}=\left(X, L, L_{0}, L_{F}, C, \Delta, \Lambda\right) \text { and } \\
& \left(Q_{a, t}^{\mathrm{sym}}, Q_{a, t, 0}^{\mathrm{sym}}, Q_{a, t, F}^{\mathrm{sym}}, \rightarrow_{a, t}^{\mathrm{sym}}, W_{a, t}^{\mathrm{sym}}\right) \text { is the WSTTS of the TSWA } \mathcal{W} \text { and } \\
& \text { the constant signal } a^{t} \text {. } \\
& \operatorname{incr}_{<}(a, t)(w)=\left\{\left(l^{\prime}, Z^{\prime}, \overline{a^{\prime}}, s^{\prime}\right) \in L \times \mathcal{Z}(C \amalg\{T\}) \times\left(\mathbb{D}^{X}\right)^{\circledast} \times S \mid \forall \nu^{\prime} \in Z^{\prime} . \nu^{\prime}(T)<t,\right. \\
& \left.s^{\prime}=\bigoplus_{(l, Z, \bar{a}, s) \in w} s \otimes \operatorname{Dist}\left(\{(l, Z, \bar{a})\},\left\{\left(l^{\prime}, Z^{\prime}, \overline{a^{\prime}}\right)\right\}, Q_{a, t}^{\mathrm{sym}}, \rightarrow_{a, t}^{\mathrm{sym}}, W_{a, t}^{\mathrm{sym}}\right)\right\}
\end{aligned}
$$

Algorithm 2 shows our online algorithm for quantitative timed pattern matching. We construct the matching automaton $\mathcal{A}_{\text {match }}$ from the TSA $\mathcal{A}$ (line 1), and we try matching by reading each constant sub-signal $a_{i}^{\tau_{i}}$ of the signal $\sigma=a_{1}^{\tau_{1}} a_{2}^{\tau_{2}} \cdots a_{n}^{\tau_{n}}$ much like the illustration in Fig. 3. For each $i$, first, we consume a prefix $a_{i}^{\tau_{i}^{\prime}}$ of $a_{i}^{\tau_{i}}=a_{i}^{\tau_{i}^{\prime}} a_{i}^{\tau_{i}^{\prime \prime}}$ and update the intermediate weight weight (line 4). Then, we update the result $M$ for each weight $(l, Z, \varepsilon, s) \in$ weight labelled with an accepting location (line 7). Finally, we consume the remaining part $a_{i}^{\tau_{i}^{\prime \prime}}$ and update the intermediate weight weight (line 8).

Complexity discussion In general, the time and space complexities of Algorithm 2 are polynomial to the length $n$ of the signal $\sigma=a_{1}^{\tau_{1}} a_{2}^{\tau_{2}} \cdots a_{n}^{\tau_{n}}$ due to the bound of the size of the reachability part of the WSTTS. On the other hand, if the TSWA has a time-bound and the sampling frequency of the signal is also bounded (such as in Figs. 6 and 7), time and space complexities are linear and constant to the length $n$ of the signal, respectively.

\section{Experiments}

We implemented our online algorithm for quantitative timed pattern matching in $\mathrm{C}++$ and conducted experiments to answer the following research questions. We suppose that the input piecewise-constant signals are interpolations of the actual signals by sampling.

RQ1 Is the practical performance of Algorithm 2 realistic?

RQ2 Is Algorithm 2 online capable, i.e., does it perform in linear time and constant space, with respect to the number of the entries in the signal?

RQ3 Can Algorithm 2 handle denser logs, i.e., what is the performance with respect to the sampling frequency of the signal?

Our implementation is in https://github.com/MasWag/qtpm. We conducted the experiments on an Amazon EC2 c4.large instance (2 vCPUs and $3.75 \mathrm{GiB}$ RAM) running Ubuntu 18.04 LTS (64 bit). We compiled the implementation by GCC-4.9.3. For the measurement of the execution time and memory usage, we 
used GNU time and took an average of 20 executions. We could not compare with [BFMU17] because their implementation is not publicly available.

As the complete semiring $\mathbb{S}$, we used the sup-inf semiring ( $\mathbb{R} \amalg$ $\{ \pm \infty\}$, sup, inf, $-\infty,+\infty)$ and the tropical semiring $(\mathbb{R} \amalg\{+\infty\}$, inf, $+,+\infty, 0)$ in Example 4 . We used the cost functions $\kappa_{r}$ in Example 8 for the sup-inf semiring, and $\kappa_{t}$ in Example 8 for the tropical semiring.

Benchmarks We used the automotive benchmark problems shown in Figs. 6 to 8. A summary of quantitative timed pattern matching is on the right of each figure. The specified behaviors in the TSWAs are taken from ST-Lib $\left[\mathrm{KJD}^{+} 16\right]$ and known to be useful for automotive control applications. See Appendix B for a performance comparison among the benchmarks.

RQ1: Practical Performance Figs. 9 and 10 show the execution time and memory usage of our quantitative timed pattern matching for the TSWAs $\mathcal{W}$ and signals $\sigma$. Here, we fixed the sampling frequency to be $0.1 \mathrm{~Hz}$ and changed the duration $|\sigma|$ of the signal from $60,000 \mathrm{~s}$ to $600,000 \mathrm{~s}$ in Overshoot and Ringing, and from $1,000 \mathrm{~s}$ to $10,000 \mathrm{~s}$ in Overshoot (UnBounded).

In Fig. 9, we observe that Algorithm 2 handles the log with 60,000 entries in less than $20 \mathrm{~s}$ with less than $7.1 \mathrm{MiB}$ of memory usage for OvERSHOOT, and in about 1 or 2 minutes with less than $7.8 \mathrm{MiB}$ of memory usage for Ringing. In Fig. 10, we observe that Algorithm 2 handles the log with 10,000 entries in less than $120 \mathrm{~s}$ with less than $250 \mathrm{MiB}$ of memory usage for Overshoot (UNBOUNDED). Although the quantitative timed pattern matching problem is complex, we conclude that its practical performance is realistic.

RQ2: Change in Speed and Memory Usage with Signal Size Figs. 9 and 10 show the execution time and memory usage of our quantitative timed pattern matching. See RQ1 for the detail of our experimental setting.

In Fig. 9, for the TSAs with time-bound, we observe that the execution time is linear with respect to the duration $|\sigma|$ of the input signals and the memory usage is more or less constant with respect to the duration $|\sigma|$ of the input signals. This performance is essential for a monitor to keep monitoring a running system.

In Fig. 10, for the TSA without any time-bound, we observe that the execution time is cubic and the memory usage is quadratic with respect to the number of the entries in $|\sigma|$. The memory usage increases quadratically with the number of the entries because the intermediate weight weight $_{j}$ has an entry for each initial interval $\left[\tau_{i}, \tau_{i+1}\right)$ of the trimming and for each interval $\left[\tau_{k}, \tau_{k+1}\right)$ where the transition occurred. The execution time increases cubically with respect to the number of the entries because the shortest distance is computed for each entry of weight $_{j}$. However, we note that our quantitative timed pattern matching still works when the number of the entries is relatively small.

RQ3: Change in Speed and Memory Usage with Sampling Frequency Fig. 11 shows the execution time and memory usage of our quantitative timed pattern matching for each TSWA $\mathcal{W}$ and signal $\sigma$ of Overshoot and Ringing. Here, we 

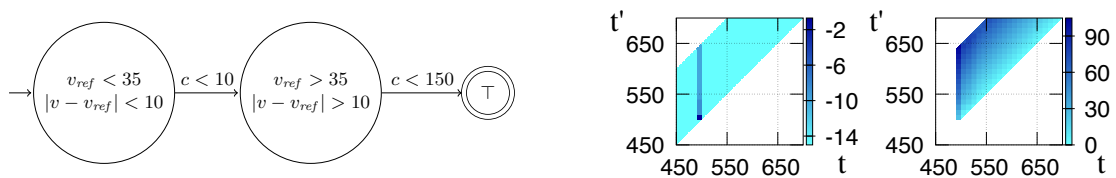

Fig. 6: Overshoot: The set of input signals is generated by the cruise control model [cru]. The TSA is for the settling when the reference value of the velocity is changed from $v_{r e f}<35$ to $v_{\text {ref }}>35$. The left and right maps are for the sup-inf and tropical semirings, respectively.
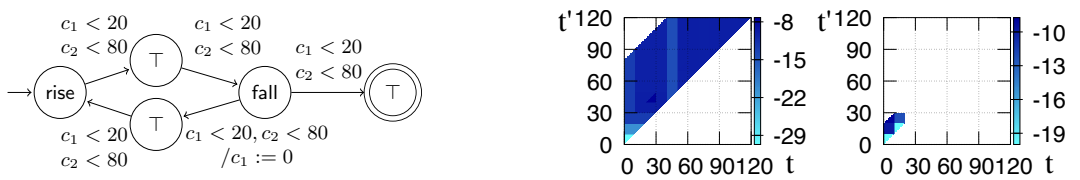

Fig. 7: Ringing: The set of input signals is generated by the same model [cru] as that in Overshoot. The TSA is for the frequent rise and fall of the signal in $80 \mathrm{~s}$. The constraints rise and fall are rise $=v(t)-v(t-10)>10$ and fall $=$ $v(t)-v(t-10)<-10$. The left and right maps are for the sup-inf and tropical semirings, respectively.
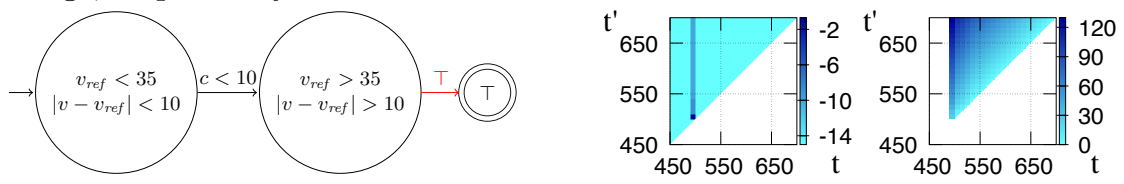

Fig. 8: Overshoot (Unbounded): The set of input signals is generated by the same model [cru] as that in Overshoot. The TSA is almost the same as that in Overshoot, but the time-bound $(c<150)$ is removed. The left and right maps are for the sup-inf and tropical semirings, respectively.
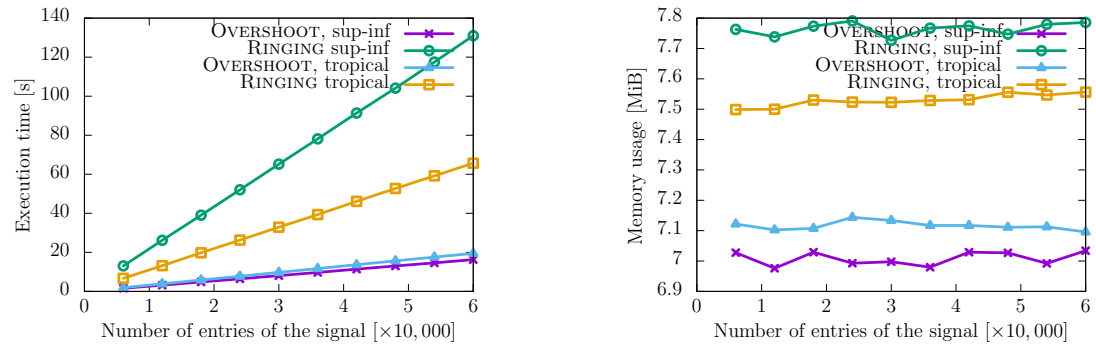

Fig. 9: Change in execution time (left) and memory usage (right) for OvERSHOOT and RINGING with the number of the entries of the signals
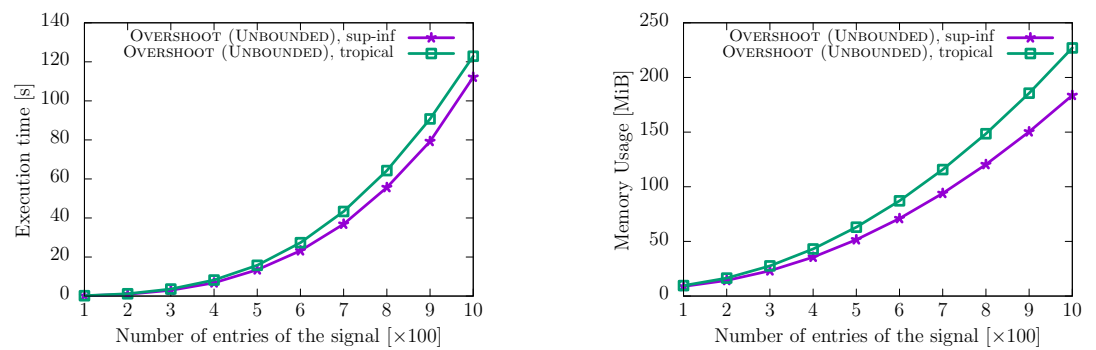

Fig. 10: Change in execution time (left) and memory usage (right) for OverSHOOT (UNBOUNDED) with the number of the entries of the signals 

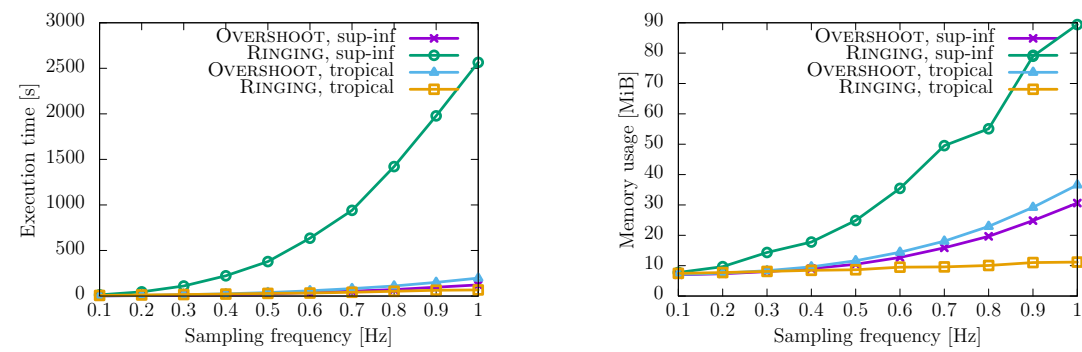

Fig. 11: Change in execution time (left) and memory usage (right) for OverSHOOT and RINGING with the sampling frequency

fixed the number of the entries to be 6,000 and changed the sampling frequency from $0.1 \mathrm{~Hz}$ to $1.0 \mathrm{~Hz}$.

In Fig. 11, we observe that the execution time is cubic, and the memory usage is more or less quadratic with respect to the sampling frequency of the signals. This is because the number of the entries in a certain duration is linear to the sampling frequency, which increases the number of the reachability states of the WSTTSs quadratically. Despite the steep curve of the execution time, we also observe that the execution time is smaller than the duration of the signal. Therefore, our algorithm is online capable at least for these sampling frequencies.

\section{Conclusions and future work}

Using an automata-based approach, we proposed an online algorithm for quantitative timed pattern matching. The key idea of this approach is the reduction to the shortest distance of a weighted graph using zones.

Comparison of the expressiveness of TSWAs with other formalisms e. g., signal temporal logic [MN04] or signal regular expressions [BFMU17] is future work. Another future work is the comparison with the quantitative semantics based on the distance between traces presented in [JBGN18]. 


\section{References}

ACM97. Eugene Asarin, Paul Caspi, and Oded Maler. A Kleene theorem for timed automata. In Proceedings, 12th Annual IEEE Symposium on Logic in Computer Science, Warsaw, Poland, June 29 - July 2, 1997, pages 160-171. IEEE Computer Society, 1997.

AD94. Rajeev Alur and David L. Dill. A theory of timed automata. Theor. Comput. Sci., 126(2):183-235, 1994.

AH15. Takumi Akazaki and Ichiro Hasuo. Time robustness in MTL and expressivity in hybrid system falsification. In Daniel Kroening and Corina S. Pasareanu, editors, Computer Aided Verification - 27th International Conference, CAV 2015, San Francisco, CA, USA, July 18-24, 2015, Proceedings, Part II, volume 9207 of Lecture Notes in Computer Science, pages 356-374. Springer, 2015.

AHW18. Étienne André, Ichiro Hasuo, and Masaki Waga. Offline timed pattern matching under uncertainty. In 23rd International Conference on Engineering of Complex Computer Systems, ICECCS 2018, Melbourne, Australia, December 12-14, 2018, pages 10-20. IEEE Computer Society, 2018.

ALFS11. Yashwanth Annpureddy, Che Liu, Georgios E. Fainekos, and Sriram Sankaranarayanan. S-taliro: A tool for temporal logic falsification for hybrid systems. In Parosh Aziz Abdulla and K. Rustan M. Leino, editors, Tools and Algorithms for the Construction and Analysis of Systems - 17th International Conference, TACAS 2011, Held as Part of the Joint European Conferences on Theory and Practice of Software, ETAPS 2011, Saarbrücken, Germany, March 26-April 3, 2011. Proceedings, volume 6605 of Lecture Notes in Computer Science, pages 254-257. Springer, 2011.

BFMU17. Alexey Bakhirkin, Thomas Ferrère, Oded Maler, and Dogan Ulus. On the quantitative semantics of regular expressions over real-valued signals. In FORMATS, volume 10419 of Lecture Notes in Computer Science, pages 189-206. Springer, 2017.

$\mathrm{BFN}^{+}$18. Alexey Bakhirkin, Thomas Ferrère, Dejan Nickovic, Oded Maler, and Eugene Asarin. Online timed pattern matching using automata. In David N. Jansen and Pavithra Prabhakar, editors, Formal Modeling and Analysis of Timed Systems - 16th International Conference, FORMATS 2018, Beijing, China, September 4-6, 2018, Proceedings, volume 11022 of Lecture Notes in Computer Science, pages 215-232. Springer, 2018.

BM15. Ezio Bartocci and Rupak Majumdar, editors. Runtime Verification - 6th International Conference, RV 2015 Vienna, Austria, September 22-25, 2015. Proceedings, volume 9333 of Lecture Notes in Computer Science. Springer, 2015.

$\mathrm{BVP}^{+}$16. Giuseppe Bombara, Cristian Ioan Vasile, Francisco Penedo, Hirotoshi Yasuoka, and Calin Belta. A decision tree approach to data classification using signal temporal logic. In Alessandro Abate and Georgios E. Fainekos, editors, Proceedings of the 19th International Conference on Hybrid Systems: Computation and Control, HSCC 2016, Vienna, Austria, April 12-14, 2016, pages 1-10. ACM, 2016.

BY03. Johan Bengtsson and Wang Yi. Timed automata: Semantics, algorithms and tools. In Jörg Desel, Wolfgang Reisig, and Grzegorz Rozenberg, editors, Lectures on Concurrency and Petri Nets, Advances in Petri Nets [This tutorial volume originates from the 4 th Advanced Course on Petri Nets, ACPN 
2003, held in Eichstätt, Germany in September 2003. In addition to lectures given at ACPN 2003, additional chapters have been commissioned], volume 3098 of Lecture Notes in Computer Science, pages 87-124. Springer, 2003.

CHO16. Krishnendu Chatterjee, Thomas A. Henzinger, and Jan Otop. Quantitative monitor automata. In Xavier Rival, editor, Static Analysis - 23rd International Symposium, SAS 2016, Edinburgh, UK, September 8-10, 2016, Proceedings, volume 9837 of Lecture Notes in Computer Science, pages 23-38. Springer, 2016.

cru. tprasadtp/cruise-control-simulink: Simulink model for Cruise control system of a car with dynamic road conditions. https://github.com/tprasadtp/ cruise-control-simulink.

CSWL16. Sanjian Chen, Oleg Sokolsky, James Weimer, and Insup Lee. Data-driven adaptive safety monitoring using virtual subjects in medical cyber-physical systems: A glucose control case study. JCSE, 10(3), 2016.

$\mathrm{DDG}^{+}$15. Jyotirmoy V. Deshmukh, Alexandre Donzé, Shromona Ghosh, Xiaoqing Jin, Garvit Juniwal, and Sanjit A. Seshia. Robust online monitoring of signal temporal logic. In Bartocci and Majumdar [BM15], pages 55-70.

Dil89. David L. Dill. Timing assumptions and verification of finite-state concurrent systems. In Joseph Sifakis, editor, Automatic Verification Methods for Finite State Systems, International Workshop, Grenoble, France, June 1214, 1989, Proceedings, volume 407 of Lecture Notes in Computer Science, pages 197-212. Springer, 1989.

DKV09. Manfred Droste, Werner Kuich, and Heiko Vogler. Handbook of Weighted Automata. Springer Publishing Company, Incorporated, 1st edition, 2009.

DM10. Alexandre Donzé and Oded Maler. Robust satisfaction of temporal logic over real-valued signals. In Krishnendu Chatterjee and Thomas A. Henzinger, editors, Formal Modeling and Analysis of Timed Systems - 8th International Conference, FORMATS 2010, Klosterneuburg, Austria, September 8-10, 2010. Proceedings, volume 6246 of Lecture Notes in Computer Science, pages 92-106. Springer, 2010.

Don10. Alexandre Donzé. Breach, A toolbox for verification and parameter synthesis of hybrid systems. In Tayssir Touili, Byron Cook, and Paul B. Jackson, editors, Computer Aided Verification, 22nd International Conference, CAV 2010, Edinburgh, UK, July 15-19, 2010. Proceedings, volume 6174 of Lecture Notes in Computer Science, pages 167-170. Springer, 2010.

FP09. Georgios E. Fainekos and George J. Pappas. Robustness of temporal logic specifications for continuous-time signals. Theor. Comput. Sci., 410(42):4262-4291, 2009.

$\mathrm{JBG}^{+}$18. Stefan Jaksic, Ezio Bartocci, Radu Grosu, Thang Nguyen, and Dejan Nickovic. Quantitative monitoring of STL with edit distance. Formal Methods in System Design, 53(1):83-112, 2018.

JBGN18. Stefan Jaksic, Ezio Bartocci, Radu Grosu, and Dejan Nickovic. An algebraic framework for runtime verification. IEEE Trans. on CAD of Integrated Circuits and Systems, 37(11):2233-2243, 2018.

KCDK15. Aaron Kane, Omar Chowdhury, Anupam Datta, and Philip Koopman. A case study on runtime monitoring of an autonomous research vehicle (ARV) system. In Bartocci and Majumdar [BM15], pages 102-117.

$\mathrm{KJD}^{+} 16$. James Kapinski, Xiaoqing Jin, Jyotirmoy Deshmukh, Alexandre Donze, Tomoya Yamaguchi, Hisahiro Ito, Tomoyuki Kaga, Shunsuke Kobuna, and Sanjit Seshia. St-lib: a library for specifying and classifying model behaviors. Technical report, SAE Technical Paper, 2016. 
MN04. Oded Maler and Dejan Nickovic. Monitoring temporal properties of continuous signals. In Yassine Lakhnech and Sergio Yovine, editors, Formal Techniques, Modelling and Analysis of Timed and Fault-Tolerant Systems, Joint International Conferences on Formal Modelling and Analysis of Timed Systems, FORMATS 2004 and Formal Techniques in Real-Time and FaultTolerant Systems, FTRTFT 2004, Grenoble, France, September 22-24, 2004, Proceedings, volume 3253 of Lecture Notes in Computer Science, pages 152166. Springer, 2004.

Moh09. Mehryar Mohri. Weighted Automata Algorithms, pages 213-254. Springer Berlin Heidelberg, Berlin, Heidelberg, 2009.

RSE08. Franco Raimondi, James Skene, and Wolfgang Emmerich. Efficient online monitoring of web-service slas. In Mary Jean Harrold and Gail C. Murphy, editors, Proceedings of the 16th ACM SIGSOFT International Symposium on Foundations of Software Engineering, 2008, Atlanta, Georgia, USA, November 9-14, 2008, pages 170-180. ACM, 2008.

Sch61. Marcel Paul Schützenberger. On the definition of a family of automata. Information and Control, 4(2-3):245-270, 1961.

UFAM14. Dogan Ulus, Thomas Ferrère, Eugene Asarin, and Oded Maler. Timed pattern matching. In Axel Legay and Marius Bozga, editors, Formal Modeling and Analysis of Timed Systems - 12th International Conference, FORMATS 2014, Florence, Italy, September 8-10, 2014. Proceedings, volume 8711 of Lecture Notes in Computer Science, pages 222-236. Springer, 2014.

UFAM16. Dogan Ulus, Thomas Ferrère, Eugene Asarin, and Oded Maler. Online timed pattern matching using derivatives. In Marsha Chechik and Jean-François Raskin, editors, Tools and Algorithms for the Construction and Analysis of Systems - 22nd International Conference, TACAS 2016, Held as Part of the European Joint Conferences on Theory and Practice of Software, ETAPS 2016, Eindhoven, The Netherlands, April 2-8, 2016, Proceedings, volume 9636 of Lecture Notes in Computer Science, pages 736-751. Springer, 2016.

Ulu17. Dogan Ulus. Montre: A tool for monitoring timed regular expressions. In Rupak Majumdar and Viktor Kuncak, editors, Computer Aided Verification - 29th International Conference, CAV 2017, Heidelberg, Germany, July 2428, 2017, Proceedings, Part I, volume 10426 of Lecture Notes in Computer Science, pages 329-335. Springer, 2017.

$\mathrm{VHL}^{+} 12$. Margus Veanes, Pieter Hooimeijer, Benjamin Livshits, David Molnar, and Nikolaj Bjørner. Symbolic finite state transducers: algorithms and applications. In John Field and Michael Hicks, editors, Proceedings of the 39th ACM SIGPLAN-SIGACT Symposium on Principles of Programming Languages, POPL 2012, Philadelphia, Pennsylvania, USA, January 22-28, 2012, pages 137-150. ACM, 2012.

WA19. Masaki Waga and Étienne André. Online parametric timed pattern matching with automata-based skipping. CoRR, abs/1903.07328, 2019.

WAH. Masaki Waga, Étienne André, and Ichiro Hasuo. Symbolic monitoring against specifications parametric in time and data. In To appear in Proc. CAV'2019.

WAH16. Masaki Waga, Takumi Akazaki, and Ichiro Hasuo. A Boyer-Moore type algorithm for timed pattern matching. In Martin Fränzle and Nicolas Markey, editors, Proceedings of the 14th International Conference on Formal Modeling and Analysis of Timed Systems (FORMATS 2016), volume 9884 of Lecture Notes in Computer Science, pages 121-139. Springer, 2016. 
WHS17. Masaki Waga, Ichiro Hasuo, and Kohei Suenaga. Efficient online timed pattern matching by automata-based skipping. In Alessandro Abate and Gilles Geeraerts, editors, Formal Modeling and Analysis of Timed Systems - 15th International Conference, FORMATS 2017, Berlin, Germany, September 57, 2017, Proceedings, volume 10419 of Lecture Notes in Computer Science, pages 224-243. Springer, 2017.

WHS18. Masaki Waga, Ichiro Hasuo, and Kohei Suenaga. MONAA: A tool for timed pattern matching with automata-based acceleration. In $3 r d$ Workshop on Monitoring and Testing of Cyber-Physical Systems, MT@CPSWeek 2018, Porto, Portugal, April 10, 2018, pages 14-15. IEEE, 2018. 


\section{A Omitted Proof}

Definition 20 (path value). For a WTTS $\mathcal{S}=\left(Q, Q_{0}, Q_{F}, \rightarrow, W\right)$, a sequence $q_{0}, q_{1}, \ldots, q_{n}$ of $Q$ is a path of $\mathcal{S}$ if we have $q_{0} \rightarrow q_{1} \rightarrow \ldots \rightarrow q_{n}$. For a WTTS $\mathcal{S}=\left(Q, Q_{0}, Q_{F}, \rightarrow, W\right)$ and a path $\pi=q_{0}, q_{1}, \ldots, q_{n}$ of $\mathcal{S}$, the path value is $\mu(\pi)=\bigotimes_{i=1}^{n} W\left(q_{i-1}, q_{i}\right)$

For any WTTS $\mathcal{S}=\left(Q, Q_{0}, Q_{F}, \rightarrow, W\right)$, we have $\alpha(\mathcal{S})=\bigoplus_{\pi \in \operatorname{ARuns}(\mathcal{S})} \mu(\pi)$, where $\operatorname{ARuns}(\mathcal{S})$ is the set of paths of $q_{0}, q_{1}, \ldots, q_{n}$ of $\mathcal{S}$ satisfying $q_{0} \in Q_{0}$ and $q_{n} \in Q_{F}$.

\section{A.1 Finiteness of the reachable part of WSTTSs}

For a WSTTS $\mathcal{S}^{\mathrm{sym}}=\left(Q^{\mathrm{sym}}, Q_{0}^{\mathrm{sym}}, Q_{F}^{\mathrm{sym}}, \rightarrow^{\mathrm{sym}}, W^{\mathrm{sym}}\right)$, we denote the reachable set by $\operatorname{Reach}\left(\mathcal{S}^{\mathrm{sym}}\right)=Q_{0}^{\mathrm{sym}} \cup\left\{q^{\mathrm{sym}} \in Q^{\text {sym }} \mid \exists q_{0}^{\text {sym }} \in\right.$ $Q_{0}^{\mathrm{sym}}, q_{1}^{\mathrm{sym}}, q_{2}^{\mathrm{sym}}, \ldots, q_{m}^{\mathrm{sym}} \in Q^{\mathrm{sym}} \cdot q_{0}^{\mathrm{sym}}, q_{1}^{\mathrm{sym}}, \ldots, q_{m}^{\mathrm{sym}}, q^{\mathrm{sym}}$ is a path of $\left.\mathcal{S}^{\mathrm{sym}}\right\}$.

Lemma 21. Let $\mathcal{S}^{\mathrm{sym}}=\left(Q^{\mathrm{sym}}, Q_{0}^{\mathrm{sym}}, Q_{F}^{\mathrm{sym}}, \rightarrow^{\mathrm{sym}}, W^{\mathrm{sym}}\right)$ be a WSTTS of a signal $\sigma=a_{1}^{\tau_{1}} a_{2}^{\tau_{2}} \cdots a_{n}^{\tau_{n}}$ and a TSWA $\mathcal{W}$. For any $(l, Z, \bar{a}) \in \operatorname{Reach}\left(\mathcal{S}^{\mathrm{sym}}\right)$, $\nu \in Z$, and $c \in C$, we have $0 \leq \nu(c) \leq|\sigma|$.

Proof. Since for any $(l, Z, \bar{a}) \in Q^{\text {sym }}$ and $\nu \in Z$, we have $\nu(T) \leq|\sigma|$, it suffice to prove $\nu(c) \leq \nu(T)$ for any $c \in C$. Let $(l, Z, \bar{a}) \in \operatorname{Reach}\left(\mathcal{S}^{\mathrm{sym}}\right)$. If $(l, Z, \bar{a}) \in Q_{0}^{\mathrm{sym}}$, we have $Z=\mathbf{0}_{C \amalg\{T\}}$ and for any $\nu \in Z$ and for any $c \in C$, we have $\nu(c)=$ $\nu(T)=0$.

Assume $(l, Z, \bar{a}) \notin Q_{0}^{\text {sym }}$ and let $\left(l^{\prime}, Z^{\prime}, \bar{a}^{\prime}\right) \in \operatorname{Reach}\left(\mathcal{S}^{\text {sym }}\right)$ satisfying $\left(\left(l^{\prime}, Z^{\prime}, \bar{a}^{\prime}\right),(l, Z, \bar{a})\right) \in \rightarrow^{\mathrm{sym}}$. If $\bar{a}=\varepsilon$, there exists $\left(l, g, \rho, l^{\prime}\right) \in \Delta$ satisfying $Z=\left\{\nu^{\prime}[\rho:=0] \mid \nu^{\prime} \in Z^{\prime}, \nu^{\prime}=g\right\}$. Since $T \notin \rho, \forall \nu^{\prime} \in Z^{\prime}, c \in C . \nu^{\prime}(c) \leq \nu^{\prime}(T)$ implies $\forall \nu \in Z, c \in C . \nu(c) \leq \nu(T)$.

If $\bar{a} \neq \varepsilon$, for any $\nu \in Z$, there are $\nu^{\prime} \in Z^{\prime}$ and $\tau \in \mathbb{R}_{>0}$ satisfying $\nu=\nu^{\prime}+\tau$. Therefore, $\forall \nu^{\prime} \in Z^{\prime}, c \in C . \nu^{\prime}(c) \leq \nu^{\prime}(T)$ implies $\forall \nu \in Z, c \in C . \nu(c) \leq \nu(T)$.

For a nonempty zone $Z \in \mathcal{Z}(C \amalg\{T\})$ and $c, c^{\prime} \in C \amalg\{T, 0\}$, we define $\prec_{Z, c, c^{\prime}} \in\{<, \leq\}$ and $d_{Z, c, c^{\prime}} \in \mathbb{R} \amalg\{\infty\}$ be the smallest elements satisfying the following, where we define $<$ is smaller than $\leq$ and we denote $\nu(0)=0$.

$$
Z=\left\{\nu \mid \bigwedge_{c, c^{\prime} \in C \amalg\{T, 0\}}\left(\nu(c)-\nu\left(c^{\prime}\right)\right) \prec_{Z, c, c^{\prime}} d_{Z, c, c^{\prime}}\right\}
$$

Lemma 22. Let $\mathcal{S}^{\mathrm{sym}}=\left(Q^{\mathrm{sym}}, Q_{0}^{\mathrm{sym}}, Q_{F}^{\mathrm{sym}}, \rightarrow^{\mathrm{sym}}, W^{\mathrm{sym}}\right)$ be a WSTTS of a signal $\sigma=a_{1}^{\tau_{1}} a_{2}^{\tau_{2}} \cdots a_{n}^{\tau_{n}}$ and a TSWA $\mathcal{W}$. For any $(l, Z, \bar{a}) \in \operatorname{Reach}\left(\mathcal{S}^{\operatorname{sym}}\right)$ and $c, c^{\prime} \in C \amalg\{T, 0\}$, we have $d_{Z, c, c^{\prime}}=\infty$ or there is $k_{i} \in \mathbb{Z}$ for each $i \in\{0,1, \ldots, n\}$ satisfying $d_{Z, c, c^{\prime}}=k_{0}+\sum_{i=1}^{n}\left(k_{i}\left(\sum_{j=1}^{i} \tau_{j}\right)\right)$.

Proof. If $(l, Z, \bar{a}) \in Q_{0}^{\mathrm{sym}}$, we have $Z=\mathbf{0}_{C \amalg\{T\}}$ and we have $d_{Z, c, c^{\prime}}=0$ for each $c, c^{\prime} \in C \amalg\{T, 0\}$. 
Assume $(l, Z, \bar{a}) \notin Q_{0}^{\text {sym }}$ and let $\left(l^{\prime}, Z^{\prime}, \bar{a}^{\prime}\right) \in \operatorname{Reach}\left(\mathcal{S}^{\text {sym }}\right)$ satisfying $\left(\left(l^{\prime}, Z^{\prime}, \bar{a}^{\prime}\right),(l, Z, \bar{a})\right) \in \rightarrow^{\mathrm{sym}}$. If $\bar{a}=\varepsilon$, there exists $\left(l, g, \rho, l^{\prime}\right) \in \Delta$ satisfying $Z=\left\{\nu^{\prime}[\rho:=0] \mid \nu^{\prime} \in Z^{\prime}, \nu^{\prime} \models g\right\}$. For each $c \in \rho$, we have $d_{Z, c, 0}=d_{Z, 0, c}=0$. For each $c, c^{\prime} \in C \amalg\{T, 0\}, d_{Z, c, c^{\prime}}$ is the shortest distance in the graph interpretation of $Z^{\prime}$, where for each $c \in \rho, d_{Z^{\prime}, c, 0}$ and $d_{Z, 0, c}$ are replaced with 0. (See e.g., [BY03] for the graph interpretation of a zone.) Therefore, for each $c, c^{\prime} \in C \amalg\{T, 0\}$, there are $k_{c^{\prime \prime}, c^{\prime \prime \prime}} \in \mathbb{Z}$ satisfying $d_{Z, c, c^{\prime}}=\sum_{c^{\prime \prime}, c^{\prime \prime \prime} \in C \amalg\{T, 0\}} k_{c^{\prime \prime}, c^{\prime \prime \prime}} d_{Z^{\prime}, c^{\prime \prime}, c^{\prime \prime \prime}}$. By induction hypothesis, For any $c, c^{\prime} \in C \amalg\{T, 0\}$, we have $d_{Z, c, c^{\prime}}=\infty$ or there is $k_{i} \in \mathbb{Z}$ for each $i \in\{0,1, \ldots, n\}$ satisfying $d_{Z, c, c^{\prime}}=k_{0}+\sum_{i=1}^{n}\left(k_{i}\left(\sum_{j=1}^{i} \tau_{j}\right)\right)$.

If $\bar{a} \neq \varepsilon, d_{Z, c, c^{\prime}}$ are computed by the following procedure.

1. For each $c \in C$, we replace $\left(d_{Z^{\prime}, c, 0}, \prec Z_{Z^{\prime}, c, 0}\right)$ with $(\infty,<)$.

2. We replace $\left(d_{Z^{\prime}, T, 0}, \prec_{Z^{\prime}, c, 0}\right)$ and $\left(d_{Z^{\prime}, 0, T}, \prec_{Z^{\prime}, 0, c}\right)$ with $\left(\sum_{j=0}^{i} \tau_{j},<\right)$ and $\left(\sum_{j=0}^{i-1} \tau_{j},<\right)$, or $\left(\sum_{j=0}^{i} \tau_{j}, \leq\right)$ and $\left(\sum_{j=0}^{i} \tau_{j}, \leq\right)$, respectively.

3. We take the shortest distance in the graph interpretation of $Z^{\prime}$, where some weights are replaced by the above.

Therefore, for each $c, c^{\prime} \in C \amalg\{T, 0\}$ and for each $i \in\{0,1, \ldots, n\}$, there are $k_{c^{\prime \prime}, c^{\prime \prime \prime}} \in \mathbb{Z}$ and $k_{i} \in \mathbb{Z}$ satisfying the following.

$$
d_{Z, c, c^{\prime}}=\sum_{c^{\prime \prime}, c^{\prime \prime \prime} \in C \amalg\{T, 0\}} k_{c^{\prime \prime}, c^{\prime \prime \prime}} d_{Z^{\prime}, c^{\prime \prime}, c^{\prime \prime \prime}}+\sum_{i=1}^{n}\left(k_{i}\left(\sum_{j=1}^{i} \tau_{j}\right)\right)
$$

By induction hypothesis, For any $c, c^{\prime} \in C \amalg\{T, 0\}$, we have $d_{Z, c, c^{\prime}}=\infty$ or there is $k_{i} \in \mathbb{Z}$ for each $i \in\{0,1, \ldots, n\}$ satisfying $d_{Z, c, c^{\prime}}=k_{0}+\sum_{i=1}^{n}\left(k_{i}\left(\sum_{j=1}^{i} \tau_{j}\right)\right)$.

Lemma 23. For any WSTTS $\mathcal{S}^{\mathrm{sym}}=\left(Q^{\mathrm{sym}}, Q_{0}^{\mathrm{sym}}, Q_{F}^{\mathrm{sym}}, \rightarrow^{\mathrm{sym}}, W^{\mathrm{sym}}\right)$ and for any $(l, Z, \bar{a}) \in \operatorname{Reach}\left(\mathcal{S}^{\mathrm{sym}}\right)$, either $\bar{a}=\varepsilon$ holds or there is $t \in \mathbb{R}_{\geq 0}$ such that for any $\nu \in Z, \bar{a}=\operatorname{Values}(\sigma([t, \nu(T))))$ holds.

Proof. Let $(l, Z, \bar{a}) \in \operatorname{Reach}\left(\mathcal{S}^{\mathrm{sym}}\right)$. If $(l, Z, \bar{a}) \in Q_{0}^{\mathrm{sym}}$, we have $\bar{a}=\varepsilon$.

If $(l, Z, \bar{a}) \notin Q_{0}^{\text {sym }}$, there is $\left(l^{\prime}, Z^{\prime}, \bar{a}^{\prime}\right) \in \operatorname{Reach}\left(\mathcal{S}^{\text {sym }}\right)$ such that $\left(\left(l^{\prime}, Z^{\prime}, \bar{a}^{\prime}\right),(l, Z, \bar{a})\right) \in \rightarrow^{\text {sym }}$. If $\bar{a} \neq \varepsilon$, we have $\bar{a}=\bar{a}^{\prime} \circ \operatorname{Values}\left(\sigma\left(\left[\nu(T), \nu^{\prime}(T)\right)\right)\right)$. By induction hypothesis, there is $\nu^{\prime} \in Z^{\prime}$ such that for any $\nu \in Z$, we have $\bar{a}=\operatorname{Values}\left(\sigma\left(\left[\nu^{\prime}(T), \nu(T)\right)\right)\right)$ or there is $t \in \mathbb{R}_{\geq 0}$ such that for any $\nu \in Z$, $\bar{a}=\operatorname{Values}(\sigma([t, \nu(T))))$ holds.

Theorem 24 (finiteness). For any WSTTS $\mathcal{S}^{\mathrm{sym}}$, there are only finitely many states reachable from $Q_{0}^{\mathrm{sym}}$.

Proof. The locations $L$ is a finite set. By Lemma 21 and Lemma 22, the number of zones appearing in Reach $\left(\mathcal{S}^{\mathrm{sym}}\right)$ is finitely many. By Lemma 23 , the subsequences $\bar{a}$ appearing in $\operatorname{Reach}\left(\mathcal{S}^{\text {sym }}\right)$ is finitely many. Therefore, $\operatorname{Reach}\left(\mathcal{S}^{\text {sym }}\right)$ is a finite set. 


\section{A.2 Proof of Theorem 15}

First, we define symbolic path value.

Definition 25 (symbolic path value). For a WSTTS $\mathcal{S}^{\text {sym }}=$ $\left(Q^{\mathrm{sym}}, Q_{0}^{\mathrm{sym}}, Q_{F}^{\mathrm{sym}}, \rightarrow^{\mathrm{sym}}, W^{\mathrm{sym}}\right)$, a sequence $q_{0}^{\mathrm{sym}}, q_{1}^{\mathrm{sym}}, \ldots, q_{n}^{\mathrm{sym}}$ of $Q^{\mathrm{sym}}$ is a path of $\mathcal{S}^{\mathrm{sym}}$ if for any $i \in\{1, \ldots, n\}$, we have $\left(q_{i-1}^{\mathrm{sym}}, q_{i}^{\mathrm{sym}}\right) \in \rightarrow^{\mathrm{sym}}$. For a WSTTS $\mathcal{S}^{\mathrm{sym}}=\left(Q^{\mathrm{sym}}, Q_{0}^{\mathrm{sym}}, Q_{F}^{\mathrm{sym}}, \rightarrow^{\mathrm{sym}}, W^{\mathrm{sym}}\right)$ and a path $\pi^{\mathrm{sym}}=q_{0}^{\mathrm{sym}}, q_{1}^{\mathrm{sym}}, \ldots q_{n}^{\mathrm{sym}}$ of $\mathcal{S}^{\mathrm{sym}}$, the symbolic path value is $\mu^{\mathrm{sym}}\left(\pi^{\mathrm{sym}}\right)=\bigotimes_{i=1}^{n} W^{\mathrm{sym}}\left(q_{i-1}^{\mathrm{sym}}, q_{i}^{\mathrm{sym}}\right)$.

Similarly to the trace value $\alpha(\mathcal{S})$, for any WSTTS $\mathcal{S}^{\text {sym }}=\left(Q^{\mathrm{sym}}, Q_{0}^{\mathrm{sym}}, Q_{F}^{\mathrm{sym}}, \rightarrow^{\mathrm{sym}}, W^{\mathrm{sym}}\right), \quad$ we have $\alpha^{\mathrm{sym}}\left(\mathcal{S}^{\mathrm{sym}}\right)=$ $\bigoplus_{\pi^{\text {sym }} \in \operatorname{ARuns}\left(\mathcal{S}^{\mathrm{sym}}\right)} \mu^{\mathrm{sym}}\left(\pi^{\mathrm{sym}}\right)$, where $\operatorname{ARuns}\left(\mathcal{S}^{\mathrm{sym}}\right)$ is the set of paths of $q_{0}^{\mathrm{sym}}, q_{1}^{\mathrm{sym}}, \ldots, q_{n}^{\mathrm{sym}}$ of $\mathcal{S}^{\mathrm{sym}}$ satisfying $q_{0}^{\mathrm{sym}} \in Q_{0}^{\mathrm{sym}}$ and $q_{n}^{\mathrm{sym}} \in Q_{F}^{\mathrm{sym}}$.

For a semiring $\mathbb{S}=\left(S, \oplus, \otimes, e_{\oplus}, e_{\otimes}\right)$, we denote the canonical order by $\preceq \subseteq$ $S \times S$, where $s \preceq s^{\prime} \Longleftrightarrow s \oplus s^{\prime}=s^{\prime}$. When $\mathbb{S}$ is idempotent, $s=s^{\prime}$ if and only of $s \preceq s^{\prime}$ and $s^{\prime} \preceq s$ because: if $s=s^{\prime}$, we have $s \oplus s^{\prime}=s^{\prime} \oplus s^{\prime}=s^{\prime}$ and $s^{\prime} \oplus s=s \oplus s=s$; and if $s \preceq s^{\prime}$ and $s^{\prime} \preceq s$, we have $s=s \oplus s^{\prime}=s^{\prime} \oplus s=s^{\prime}$.

For simplicity, we assume that for any signal $\sigma=a_{1}^{\tau_{1}} a_{2}^{\tau_{2}} \ldots a_{n}^{\tau_{n}} \in \mathcal{T}\left(\mathbb{D}^{X}\right)$ and for any $i \in\{1,2, \ldots, n-1\}$, we have $a_{i} \neq a_{i+1}$.

Lemma 26. Let $\sigma=a_{1}^{\tau_{1}} a_{2}^{\tau_{2}} \cdots a_{n}^{\tau_{n}} \in \mathcal{T}\left(\mathbb{D}^{X}\right)$ be a signal, let $\mathcal{W}$ be a TSWA, and let $\mathcal{S}^{\mathrm{sym}}=\left(Q^{\mathrm{sym}}, Q_{0}^{\mathrm{sym}}, Q_{F}^{\mathrm{sym}}, \rightarrow^{\mathrm{sym}}, W^{\mathrm{sym}}\right)$ be a WSTTS of $\sigma$ and $\mathcal{W}$. For any $\left(l, Z_{1}, \bar{a}\right),\left(l^{\prime}, Z_{1}^{\prime}, \overline{a^{\prime}}\right) \in Q^{\mathrm{sym}}$ if $\left(\left(l, Z_{1}, \bar{a}\right),\left(l^{\prime}, Z_{1}^{\prime}, \overline{a^{\prime}}\right)\right) \in \rightarrow^{\mathrm{sym}}$ holds, for any $\left(l, Z_{2}, \bar{a}\right) \in Q^{\mathrm{sym}}$ satisfying $Z_{1} \subseteq Z_{2}$, there exists $\left(l^{\prime}, Z_{2}^{\prime}, \overline{a^{\prime}}\right) \in Q^{\mathrm{sym}}$ satisfying $Z_{1}^{\prime} \subseteq Z_{2}^{\prime}$ and $\left(\left(l, Z_{2}, \bar{a}\right),\left(l^{\prime}, Z_{2}^{\prime}, \overline{a^{\prime}}\right)\right) \in \rightarrow^{\mathrm{sym}}$.

Proof. If $\overline{a^{\prime}}=\varepsilon$, there exists $\left(l, g, \rho, l^{\prime}\right) \in \Delta$ satisfying $Z_{1}^{\prime}=\{\nu[\rho:=0] \mid \nu \in$ $\left.Z_{1}, \nu \models g\right\}$. Since $Z_{1} \subseteq Z_{2}, Z_{2}^{\prime}=\left\{\nu[\rho:=0] \mid \nu \in Z_{2}, \nu \models g\right\}$ is nonempty and we have $\left(\left(l, Z_{2}, \bar{a}\right),\left(l^{\prime}, Z_{2}^{\prime}, \overline{a^{\prime}}\right)\right) \in \rightarrow^{\text {sym }}$. We also have $Z_{1}^{\prime} \subseteq Z_{2}^{\prime}$.

If $\overline{a^{\prime}} \neq \varepsilon$, let $M$ be either $M_{i,=}=\left\{\nu \mid \nu(T)=\sum_{j=0}^{i} \tau_{j}\right\}$ or $M_{i}=\{\nu \mid$ $\left.\sum_{j=0}^{i-1} \tau_{j}<\nu(T)<\sum_{j=0}^{i} \tau_{j}\right\}$ satisfying $Z_{1}^{\prime}=\left\{\nu+\tau \mid \nu+Z_{1}, \tau \in \mathbb{R}_{>0}\right\} \cap M$, where $i \in\{1,2, \ldots, n\}$. Let $Z_{2}^{\prime}=\left\{\nu+\tau \mid \nu \in Z_{2}, \tau \in \mathbb{R}_{>0}\right\} \cap M$. Then, we have $\left(\left(l, Z_{2}, \bar{a}\right),\left(l^{\prime}, Z_{2}^{\prime}, \overline{a^{\prime}}\right)\right) \in \rightarrow^{\text {sym }}$ and since $Z_{1} \subseteq Z_{2}$, we have $Z_{1}^{\prime} \subseteq Z_{2}^{\prime}$.

Lemma 27. Let $\sigma=a_{1}^{\tau_{1}} a_{2}^{\tau_{2}} \cdots a_{n}^{\tau_{n}} \in \mathcal{T}\left(\mathbb{D}^{X}\right)$ be a signal and let $\mathcal{W}=(\mathcal{A}, \kappa)$ be a TSWA, where $\mathcal{A}=\left(X, L, L_{0}, L_{F}, C, \Delta, \Lambda\right)$. Let $\mathcal{S}$ and $\mathcal{S}^{\text {sym }}$ be the WTTS and $W S T T S$ of $\sigma$ and $\mathcal{W}$, respectively. For any $(l, \nu, t, \bar{a}) \rightarrow\left(l^{\prime}, \nu^{\prime}, t^{\prime}, \overline{a^{\prime}}\right)$, there is a zone $Z^{\prime} \in \mathcal{Z}(C \amalg\{T\})$ satisfying the following.

- $\left(\left(l,\left\{\nu_{Z}\right\}, \bar{a}\right),\left(l^{\prime}, Z^{\prime}, \overline{a^{\prime}}\right)\right) \in \rightarrow^{\mathrm{sym}}$, where $\nu_{Z} \in\left(\mathbb{R}_{\geq 0}\right)^{C \amalg\{T\}}$ is for any $c \in C$, $\nu_{Z}(c)=\nu(c)$ and $\nu_{Z}(T)=t$.

- There exists $\nu_{Z}^{\prime} \in Z^{\prime}$ such that for any $c \in C \nu_{Z}^{\prime}(c)=\nu^{\prime}(c)$ and $\nu_{Z}^{\prime}(T)=t^{\prime}$.

- $W\left(\left((l, \nu, t, \bar{a}),\left(l^{\prime}, \nu^{\prime}, t^{\prime}, \overline{a^{\prime}}\right)\right)\right)=W^{\operatorname{sym}}\left(\left(\left(l,\left\{\nu_{Z}\right\}, \bar{a}\right),\left(l^{\prime}, Z^{\prime}, \overline{a^{\prime}}\right)\right)\right)$ 
Proof. If $\overline{a^{\prime}}=\varepsilon$, there is $\left(l, g, \rho, l^{\prime}\right) \in \Delta$ satisfying $\nu \models g, \nu^{\prime}=\nu[\rho:=0]$, $t^{\prime}=t, \bar{a} \neq \varepsilon$, and $\overline{a^{\prime}}=\varepsilon$. Let $Z^{\prime}$ be $Z^{\prime}=\left\{\nu_{Z}[\rho:=0]\right\}$. Since $\nu_{Z} \models g, \bar{a} \neq \varepsilon$, and $\overline{a^{\prime}}=\varepsilon$, we have $\left(\left(l,\left\{\nu_{Z}\right\}, \bar{a}\right),\left(l^{\prime}, Z^{\prime}, \overline{a^{\prime}}\right)\right)$. Since $\nu^{\prime}=\nu[\rho:=0]$, for any $c \in C$, we have $\left(\nu_{Z}[\rho:=0]\right)(c)=\nu^{\prime}(c)$. Since $t=t^{\prime}$ and $T \notin \rho$, we have $\left(\nu_{Z}[\rho:=0]\right)(T)=\nu_{Z}(T)=t=t^{\prime}$. We also have $W\left(\left((l, \nu, t, \bar{a}),\left(l^{\prime}, \nu^{\prime}, t^{\prime}, \overline{a^{\prime}}\right)\right)\right)=$ $\kappa(\Lambda(l), \bar{a})=W^{\operatorname{sym}}\left(\left(\left(l,\left\{\nu_{Z}\right\}, \bar{a}\right),\left(l^{\prime}, Z^{\prime}, \overline{a^{\prime}}\right)\right)\right)$.

If $\overline{a^{\prime}} \neq \varepsilon, l=l^{\prime}$ and there is $\tau \in \mathbb{R}_{>0}$ satisfying $\nu^{\prime}=\nu+\tau, t^{\prime}=t+\tau$, and $\overline{a^{\prime}}=\bar{a} \circ \sigma([t, t+\tau))$. Let $M$ be either $M_{i,=}=\left\{\nu \mid \nu(T)=\sum_{j=0}^{i} \tau_{j}\right\}$ or $M_{i}=\left\{\nu \mid \sum_{j=0}^{i-1} \tau_{j}<\nu(T)<\sum_{j=0}^{i} \tau_{j}\right\}$ satisfying $t^{\prime} \in M$, where $i \in\{1,2, \ldots, n\}$. Let $Z^{\prime}=\left\{\nu+\tau \mid \nu \in Z, \tau \in \mathbb{R}_{>0}\right\} \cap M$. We have $\left(\left(l,\left\{\nu_{Z}\right\}, \bar{a}\right),\left(l^{\prime}, Z^{\prime}, \overline{a^{\prime}}\right)\right) \in \rightarrow^{\mathrm{sym}}$. Since $\nu^{\prime}=\nu+\tau$ and $t^{\prime} \in M$, there exists $\nu_{Z}^{\prime} \in Z^{\prime}$ such that for any $c \in C$ $\nu_{Z}^{\prime}(c)=\nu^{\prime}(c)$ and $\nu_{Z}^{\prime}(T)=t^{\prime}$. We also have $W\left(\left((l, \nu, t, \bar{a}),\left(l^{\prime}, \nu^{\prime}, t^{\prime}, \overline{a^{\prime}}\right)\right)\right)=e_{\otimes}=$ $W^{\operatorname{sym}}\left(\left(\left(l,\left\{\nu_{Z}\right\}, \bar{a}\right),\left(l^{\prime}, Z^{\prime}, \overline{a^{\prime}}\right)\right)\right)$.

Lemma 28. Let $\sigma \in \mathcal{T}\left(\mathbb{D}^{X}\right)$ be a signal and let $\mathcal{W}=(\mathcal{A}, \kappa)$ be a TSWA, where $\mathcal{A}=\left(X, L, L_{0}, L_{F}, C, \Delta, \Lambda\right)$. Let $\mathcal{S}$ and $\mathcal{S}^{\mathrm{sym}}$ be the WTTS and WSTTS of $\sigma$ and $\mathcal{W}$, respectively. For any path $\pi=\left(l_{0}, \nu_{0}, t_{0}, \overline{a_{0}}\right),\left(l_{1}, \nu_{1}, t_{1}, \overline{a_{1}}\right), \ldots,\left(l_{n}, \nu_{n}, t_{n}, \overline{a_{n}}\right)$ of $\mathcal{S}$, there is a path $\pi^{\mathrm{sym}}=\left(l_{0}, Z_{0}, \overline{a_{0}}\right),\left(l_{1}, Z_{1}, \overline{a_{1}}\right), \ldots,\left(l_{n}, Z_{n}, \overline{a_{n}}\right)$ of $\mathcal{S}$, such that $Z_{0}=\left\{\nu_{Z, 0} \mid \forall c \in C \cdot \nu_{Z, 0}(c)=\nu_{0}(c), \nu_{Z, 0}(T)=t_{0}\right\}$ and for any $i \in\{1,2, \ldots, n\}$, there exists $\nu_{Z, i} \in Z_{i}$ satisfying $\nu_{Z, i}(c)=\nu_{i}(c)$ for any $c \in C$ and $\nu_{Z, i}(T)=t_{i}$.

Proof. We prove the lemma by induction on $n$.

When $n=1$, by Lemma 27 , for $Z_{0}=\left\{\nu_{Z, 0} \in\left(\mathbb{R}_{\geq 0}\right)^{C \amalg\{T\}} \mid \forall c \in C \cdot \nu_{Z, 0}(c)=\right.$ $\left.\nu_{0}(c), \nu_{Z, 0}(T)=t_{0}\right\}$ there is a zone $Z_{1} \in \mathcal{Z}(C \amalg\{T\})$ satisfying:

- $\left(\left(l_{0}, Z_{0}, \overline{a_{0}}\right),\left(l_{1}, Z_{1}, \overline{a_{1}}\right)\right) \in \rightarrow^{\mathrm{sym}} ;$ and

- there exists $\nu_{Z, 1} \in Z_{1}$ satisfying $\nu_{Z, 1}(c)=\nu_{1}(c)$ for any $c \in C$ and $\nu_{Z, 1}(T)=$ $t_{1}$.

When $n>1$, by Lemma 27, for $Z_{n-1}^{\prime}=\left\{\nu_{Z, n-1} \in\left(\mathbb{R}_{\geq 0}\right)^{C \amalg\{T\}} \mid \forall c \in\right.$ $\left.C . \nu_{Z, n-1}(c)=\nu_{n-1}(c), \nu_{Z, n-1}(T)=t_{n-1}\right\}$ there is a zone $Z_{n}^{\prime} \in \mathcal{Z}(C \amalg\{T\})$ satisfying:

- $\left(\left(l_{n-1}, Z_{n-1}^{\prime}, \overline{a_{n-1}}\right),\left(l_{n}, Z_{n}^{\prime}, \overline{a_{n}}\right)\right) \in \rightarrow^{\mathrm{sym}} ;$ and

- there exists $\nu_{Z, n} \in Z_{n}^{\prime}$ satisfying $\nu_{Z, n}(c)=\nu_{n}(c)$ for any $c \in C$ and $\nu_{Z, n}(T)=$ $t_{n}$.

By induction hypothesis, there is a path $\left(l_{0}, Z_{0}, \overline{a_{0}}\right),\left(l_{1}, Z_{1}, \overline{a_{1}}\right), \ldots,\left(l_{n-1}, Z_{n-1}, \overline{a_{n-1}}\right)$ of $\mathcal{S}^{\mathrm{sym}}$, such that $Z_{0}=\left\{\nu_{Z, 0} \mid\right.$ $\left.\forall c \in C . \nu_{Z, 0}(c)=\nu_{0}(c), \nu_{Z, 0}(T)=t_{0}\right\}$ and for any $i \in\{1,2, \ldots, n-1\}$, there exists $\nu_{Z, i} \in Z_{i}$ satisfying $\nu_{Z, i}(c)=\nu_{i}(c)$ for any $c \in C$ and $\nu_{Z, i}(T)=t_{i}$. Since $Z_{n-1}^{\prime} \subseteq Z_{n-1}$ and Lemma 26 , there exists $Z_{n} \in \mathcal{Z}(C \amalg\{T\})$ satisfying $Z_{n}^{\prime} \subseteq Z_{n}$ and $\left(\left(l_{n-1}, Z_{n-1}, \overline{a_{n-1}}\right),\left(l_{n}, Z_{n}, \overline{a_{n}}\right)\right) \in \rightarrow^{\text {sym }}$. Therefore, $\left(l_{0}, Z_{0}, \overline{a_{0}}\right),\left(l_{1}, Z_{1}, \overline{a_{1}}\right), \ldots,\left(l_{n}, Z_{n}, \overline{a_{n}}\right)$ is a path of $\mathcal{S}^{\text {sym }}$, such that $Z_{0}=\left\{\nu_{Z, 0} \mid \forall c \in C . \nu_{Z, 0}(c)=\nu_{0}(c), \nu_{Z, 0}(T)=t_{0}\right\}$ and for any $i \in\{1,2, \ldots, n\}$, there exists $\nu_{Z, i} \in Z_{i}$ satisfying $\nu_{Z, i}(c)=\nu_{i}(c)$ for any $c \in C$ and $\nu_{Z, i}(T)=t_{i}$. 
Lemma 29. Let $\sigma \in \mathcal{T}\left(\mathbb{D}^{X}\right)$ be a signal and let $\mathcal{W}=(\mathcal{A}, \kappa)$ be a TSWA, where $\mathcal{A}=\left(X, L, L_{0}, L_{F}, C, \Delta, \Lambda\right)$. Let $\mathcal{S}$ and $\mathcal{S}^{\text {sym }}$ be the WTTS and WSTTS of $\sigma$ and $\mathcal{W}$, respectively. For any path $\pi$ of $\mathcal{S}$, there is a path $\pi^{\text {sym }}$ of $\mathcal{S}^{\text {sym }}$ satisfying $\mu(\pi)=\mu^{\mathrm{sym}}\left(\pi^{\mathrm{sym}}\right)$. Moreover, for any $\pi \in \operatorname{ARuns}(\mathcal{S})$, there is $\pi^{\mathrm{sym}} \in \operatorname{ARuns}\left(\mathcal{S}^{\mathrm{sym}}\right)$ satisfying $\mu(\pi)=\mu^{\mathrm{sym}}\left(\pi^{\mathrm{sym}}\right)$.

Proof. By Lemma 28, for any path $\pi$ = $\left(l_{0}, \nu_{0}, t_{0}, \overline{a_{0}}\right),\left(l_{1}, \nu_{1}, t_{1}, \overline{a_{1}}\right), \ldots,\left(l_{n}, \nu_{n}, t_{n}, \overline{a_{n}}\right) \quad$ of $\mathcal{S}, \quad$ there $\quad$ is a path $\pi^{\mathrm{sym}}=\left(l_{0}, Z_{0}, \overline{a_{0}}\right),\left(l_{1}, Z_{1}, \overline{a_{1}}\right), \ldots,\left(l_{n}, Z_{n}, \overline{a_{n}}\right)$ of $\mathcal{S}$. For any $i \in\{1,2, \ldots, n\}$, we have

$$
\begin{aligned}
& W\left(\left(l_{i-1}, \nu_{i-1}, t_{i-1}, \overline{a_{i-1}}\right),\left(l_{i}, \nu_{i}, t_{i}, \overline{a_{i}}\right)\right) \\
= & \begin{cases}\kappa\left(\Lambda\left(l_{i-1}, \overline{a_{i-1}}\right)\right) & \text { if } \overline{a_{i}}=\varepsilon \\
e_{\otimes} & \text { if } \overline{a_{i}} \neq \varepsilon\end{cases} \\
= & W^{\text {sym }}\left(\left(l_{i-1}, Z_{i-1}, \overline{a_{i-1}}\right),\left(l_{i}, Z_{i}, \overline{a_{i}}\right)\right)
\end{aligned}
$$

Therefore, we have $\mu(\pi)=\mu^{\mathrm{sym}}\left(\pi^{\mathrm{sym}}\right)$.

When $\pi \in \operatorname{ARuns}(\mathcal{S})$, we have $l_{0} \in L_{0}, \nu_{0}=\mathbf{0}_{C}, t_{0}=0, \overline{a_{0}}=\varepsilon, l_{n} \in L_{F}$, $t_{n}=|\sigma|$, and $\overline{a_{n}}=\varepsilon$. By Lemma 28, we have $Z_{0}=\left\{\mathbf{0}_{C \amalg\{T\}}\right\}$ and there is $\nu_{Z, n} \in Z_{n}$ satisfying $\nu_{Z, n}(T)=t_{n}$. Therefore $\pi^{\text {sym }} \in \operatorname{ARuns}\left(\mathcal{S}^{\text {sym }}\right)$ also holds.

Theorem 30. Let $\sigma \in \mathcal{T}\left(\mathbb{D}^{X}\right)$ be a signal and let $\mathcal{W}=(\mathcal{A}, \kappa)$ be a TSWA, where $\mathcal{A}=\left(X, L, L_{0}, L_{F}, C, \Delta, \Lambda\right)$. Let $\mathcal{S}$ and $\mathcal{S}^{\text {sym }}$ be the WTTS and WSTTS of $\sigma$ and $\mathcal{W}$, respectively. If the semiring $\mathbb{S}$ is idempotent, we have $\alpha(\mathcal{S}) \preceq \alpha^{\mathrm{sym}}\left(\mathcal{S}^{\mathrm{sym}}\right)$.

Proof. By Lemma 29 , there is a mapping $f: \operatorname{ARuns}(\mathcal{S}) \rightarrow \operatorname{ARuns}\left(\mathcal{S}^{\text {sym }}\right)$ satisfying $W^{\text {sym }}(f(\pi))=W(\pi)$. We have

$$
\begin{aligned}
& \alpha(\mathcal{S}) \oplus \alpha^{\mathrm{sym}}\left(\mathcal{S}^{\mathrm{sym}}\right) \\
= & \left(\bigoplus_{\pi \in A \operatorname{Runs}(\mathcal{S})} \mu(\pi)\right) \oplus\left(\bigoplus_{\pi^{\mathrm{sym}} \in A \operatorname{Runs}\left(\mathcal{S}^{\mathrm{sym}}\right)} \mu^{\mathrm{sym}}\left(\pi^{\mathrm{sym}}\right)\right) \\
= & \left(\bigoplus_{\pi^{\mathrm{sym}} \in f(A \operatorname{Runs}(\mathcal{S}))} \mu^{\mathrm{sym}}\left(\pi^{\mathrm{sym}}\right)\right) \oplus\left(\bigoplus_{\pi^{\mathrm{sym}} \in A \operatorname{Runs}\left(\mathcal{S}^{\mathrm{sym}}\right)} \mu^{\mathrm{sym}}\left(\pi^{\mathrm{sym}}\right)\right) \\
= & \left(\bigoplus_{\pi^{\mathrm{sym}} \in A \operatorname{Runs}\left(\mathcal{S}^{\mathrm{sym}}\right)} \mu^{\mathrm{sym}}\left(\pi^{\mathrm{sym}}\right)\right)=\alpha^{\mathrm{sym}}\left(\mathcal{S}^{\mathrm{sym}}\right)
\end{aligned}
$$

Therefore, we have $\alpha(\mathcal{S}) \preceq \alpha^{\text {sym }}\left(\mathcal{S}^{\text {sym }}\right)$.

Lemma 31. Let $\sigma \in \mathcal{T}\left(\mathbb{D}^{X}\right)$ be a signal and let $\mathcal{W}=(\mathcal{A}, \kappa)$ be a TSWA, where $\mathcal{A}=\left(X, L, L_{0}, L_{F}, C, \Delta, \Lambda\right)$. Let $\mathcal{S}$ and $\mathcal{S}^{\mathrm{sym}}$ be the WTTS and WSTTS of $\sigma$ and $\mathcal{W}$, respectively. For any $\left((l, Z, \bar{a}),\left(l^{\prime}, Z^{\prime}, \overline{a^{\prime}}\right)\right) \in \rightarrow^{\mathrm{sym}}$, and $\nu_{Z}^{\prime} \in Z^{\prime}$, there is a clock valuation $\nu_{Z} \in Z$ satisfying the following. 
- $\left(l, \nu_{Z} \downarrow_{C}, \nu_{Z}(T), \bar{a}\right) \rightarrow\left(l^{\prime}, \nu_{Z}^{\prime} \downarrow_{C}, \nu_{Z}^{\prime}(T), \overline{a^{\prime}}\right)$, where $\nu_{Z} \downarrow_{C}, \nu_{Z}^{\prime} \downarrow_{C} \in\left(\mathbb{R}_{\geq 0}\right)^{C}$ are for any $c \in C, \nu_{Z} \downarrow_{C}(c)=\nu_{Z}(c)$ and $\nu_{Z}^{\prime} \downarrow_{C}(c)=\nu_{Z}^{\prime}(c)$.

- $W\left(\left(\left(l, \nu_{Z} \downarrow_{C}, \nu_{Z}(T), \bar{a}\right),\left(l^{\prime}, \nu_{Z}^{\prime} \downarrow_{C}, \nu_{Z}^{\prime}(T), \bar{a}^{\prime}\right)\right)\right)=W^{\text {sym }}\left(\left((l, Z, \bar{a}),\left(l^{\prime}, Z^{\prime}, \overline{a^{\prime}}\right)\right)\right)$

Proof. If $\overline{a^{\prime}}=\varepsilon$, we have $\bar{a} \neq \varepsilon$ and there is $\left(l, g, \rho, l^{\prime}\right) \in \Delta$ satisfying $Z^{\prime}=$ $\{\nu[\rho:=0] \mid \nu \in Z, \nu \models g\}$, which is nonempty. Because of $Z^{\prime}=\{\nu[\rho:=0] \mid \nu \in$ $Z, \nu \mid=g\}$, there exists $\nu_{Z} \in Z$ such that $\nu_{Z}^{\prime}=\nu_{Z}[\rho:=0]$. Since $\nu_{Z} \downarrow_{C} \models g$, we have $\left(l, \nu_{Z} \downarrow_{C}, \nu_{Z}(T), \bar{a}\right) \rightarrow\left(l^{\prime}, \nu_{Z}^{\prime} \downarrow_{C}, \nu_{Z}^{\prime}(T), \bar{a}^{\prime}\right)$. We also have the following.

$W\left(\left(\left(l, \nu_{Z} \downarrow_{C}, \nu_{Z}(T), \bar{a}\right),\left(l^{\prime}, \nu_{Z}^{\prime} \downarrow_{C}, \nu_{Z}^{\prime}(T), \overline{a^{\prime}}\right)\right)\right)=\kappa(\Lambda(l), \bar{a})=W^{\operatorname{sym}}\left(\left((l, Z, \bar{a}),\left(l^{\prime}, Z^{\prime}, \overline{a^{\prime}}\right)\right)\right)$

If $\overline{a^{\prime}} \neq \varepsilon$, we have $l=l^{\prime}, \overline{a^{\prime}}=\bar{a} \circ \sigma\left(\left[\nu_{Z}(T), \nu_{Z}^{\prime}(T)\right)\right)$, and for any $\nu^{\prime} \in Z^{\prime}$, there exists $\nu \in Z$ and $\tau \in \mathbb{R}_{>0}$ satisfying $\nu^{\prime}=\nu+\tau$, where $\nu_{Z} \in Z$. Let $\nu_{Z} \in Z$ and $\tau \in \mathbb{R}_{>0}$ be such that $\nu_{Z}^{\prime}=\nu_{Z}+\tau$. Because of

- $l=l^{\prime}$

- $\nu_{Z}^{\prime} \downarrow_{C}=\nu_{Z} \downarrow_{C}+\tau$,

- $\nu_{Z}^{\prime}(T)=\nu_{Z}(T)+\tau$, and

- $\bar{a}^{\prime}=\bar{a} \circ \sigma\left(\left[\nu_{Z}(T), \nu_{Z}^{\prime}(T)\right)\right)$,

we have $\left(l, \nu_{Z} \downarrow_{C}, \nu_{Z}(T), \bar{a}\right) \rightarrow\left(l^{\prime}, \nu_{Z}^{\prime} \downarrow_{C}, \nu_{Z}^{\prime}(T), \overline{a^{\prime}}\right)$. Thus we also have

$W\left(\left(\left(l, \nu_{Z} \downarrow_{C}, \nu_{Z}(T), \bar{a}\right),\left(l^{\prime}, \nu_{Z}^{\prime} \downarrow_{C}, \nu_{Z}^{\prime}(T), \overline{a^{\prime}}\right)\right)\right)=e_{\otimes}=W^{\mathrm{sym}}\left(\left((l, Z, \bar{a}),\left(l^{\prime}, Z^{\prime}, \overline{a^{\prime}}\right)\right)\right)$.

Lemma 32. Let $\sigma \in \mathcal{T}\left(\mathbb{D}^{X}\right)$ be a signal and let $\mathcal{W}=(\mathcal{A}, \kappa)$ be a TSWA, where $\mathcal{A}=\left(X, L, L_{0}, L_{F}, C, \Delta, \Lambda\right)$. Let $\mathcal{S}$ and $\mathcal{S}^{\text {sym }}$ be the WTTS and WSTTS of $\sigma$ and $\mathcal{W}$, respectively. For any path $\pi^{\mathrm{sym}}=$ $\left(l_{0}, Z_{0}, \overline{a_{0}}\right),\left(l_{1}, Z_{1}, \overline{a_{1}}\right), \ldots,\left(l_{n}, Z_{n}, \overline{a_{n}}\right)$ of $\mathcal{S}$ and for any $\nu_{Z, n} \in Z_{n}$, there is a path $\pi=\left(l_{0}, \nu_{0}, t_{0}, \overline{a_{0}}\right),\left(l_{1}, \nu_{1}, t_{1}, \overline{a_{1}}\right), \ldots,\left(l_{n}, \nu_{n}, t_{n}, \overline{a_{n}}\right)$ of $\mathcal{S}$ such that we have $\left(\nu_{Z, n}\right) \downarrow_{C}=\nu_{n}$ and $\nu_{Z, n}(T)=t_{n}$, and for any $i \in\{0,1, \ldots, n-1\}$, there exists $\nu_{Z, i} \in Z_{i}$ satisfying $\left(\nu_{Z, i}\right) \downarrow_{C}=\nu_{i}$ and $\nu_{Z, i}(T)=t_{i}$.

Proof. We prove the lemma by induction on $n$. When $n=1$, by Lemma 31, for any $\nu_{Z, 1} \in Z_{1}$, there is $\nu_{Z, 0} \in Z_{0}$ satisfying $\left(l,\left(\nu_{Z, 0}\right) \downarrow_{C}, \nu_{Z, 0}(T), \bar{a}\right) \rightarrow$ $\left(l^{\prime},\left(\nu_{Z, 1}\right) \downarrow_{C}, \nu_{Z, 1}(T), \overline{a^{\prime}}\right)$.

When $n>1$, by induction hypothesis, for any $\nu_{Z, n} \in Z_{n}$, there is a path $\left(l_{1}, \nu_{1}, t_{1}, \overline{a_{1}}\right),\left(l_{2}, \nu_{2}, t_{2}, \overline{a_{2}}\right), \ldots,\left(l_{n}, \nu_{n}, t_{n}, \overline{a_{n}}\right)$ of $\mathcal{S}$, such that we have $\left(\nu_{Z, n}\right) \downarrow_{C}=\nu_{n}$ and $\nu_{Z, n}(T)=t_{n}$, and for any $i \in\{1,2, \ldots, n-1\}$, there exists $\nu_{Z, i} \in Z_{i}$ satisfying $\left(\nu_{Z, i}\right) \downarrow_{C}=\nu_{i}$ and $\nu_{Z, i}(T)=t_{i}$. By Lemma 31, there is a clock valuation $\nu_{Z, 0} \in Z_{0}$ satisfying $\left(l,\left(\nu_{Z, 0}\right) \downarrow_{C}, \nu_{Z, 0}(T), \bar{a}\right) \rightarrow\left(l^{\prime}, \nu_{1}, t_{1}, \overline{a^{\prime}}\right)$. Therefore, $\left(l_{0}, \nu_{Z, 0}, \nu_{Z, 0}(T), \overline{a_{0}}\right),\left(l_{1}, \nu_{1}, t_{1}, \overline{a_{1}}\right), \ldots,\left(l_{n}, \nu_{n}, t_{n}, \overline{a_{n}}\right)$ is a path of $\mathcal{S}$, such that we have $\left(\nu_{Z, n}\right) \downarrow_{C}=\nu_{n}$ and $\nu_{Z, n}(T)=t_{n}$, and for any $i \in\{1,2, \ldots, n-1\}$, there exists $\nu_{Z, i} \in Z_{i}$ satisfying $\left(\nu_{Z, i}\right) \downarrow_{C}=\nu_{i}$ and $\nu_{Z, i}(T)=t_{i}$.

Lemma 33. Let $\sigma \in \mathcal{T}\left(\mathbb{D}^{X}\right)$ be a signal and let $\mathcal{W}=(\mathcal{A}, \kappa)$ be a TSWA, where $\mathcal{A}=\left(X, L, L_{0}, L_{F}, C, \Delta, \Lambda\right)$. Let $\mathcal{S}$ and $\mathcal{S}^{\mathrm{sym}}$ be the WTTS and WSTTS of $\sigma$ and $\mathcal{W}$, respectively. For any path $\pi^{\mathrm{sym}}$ of $\mathcal{S}^{\mathrm{sym}}$, there is a path $\pi$ of $\mathcal{S}$ satisfying $\mu(\pi)=\mu^{\mathrm{sym}}\left(\pi^{\mathrm{sym}}\right)$. Moreover, for any $\pi^{\mathrm{sym}} \in \operatorname{ARuns}\left(\mathcal{S}^{\mathrm{sym}}\right)$, there is $\pi \in \operatorname{ARuns}(\mathcal{S})$ satisfying $\mu(\pi)=\mu^{\mathrm{sym}}\left(\pi^{\mathrm{sym}}\right)$. 
Proof. By Lemma 32, for any path

$$
\pi^{\mathrm{sym}}=\left(l_{0}, Z_{0}, \overline{a_{0}}\right),\left(l_{1}, Z_{1}, \overline{a_{1}}\right), \ldots,\left(l_{n}, Z_{n}, \overline{a_{n}}\right)
$$

of $\mathcal{S}^{\text {sym }}$. there is a path

$$
\pi=\left(l_{0}, \nu_{0}, t_{0}, \overline{a_{0}}\right),\left(l_{1}, \nu_{1}, t_{1}, \overline{a_{1}}\right), \ldots,\left(l_{n}, \nu_{n}, t_{n}, \overline{a_{n}}\right)
$$

of $\mathcal{S}$. For any $i \in\{1,2, \ldots, n\}$, we have

$$
\begin{aligned}
& W\left(\left(l_{i-1}, \nu_{i-1}, t_{i-1}, \overline{a_{i-1}}\right),\left(l_{i}, \nu_{i}, t_{i}, \overline{a_{i}}\right)\right) \\
= & \begin{cases}\kappa\left(\Lambda\left(l_{i-1}, \overline{a_{i-1}}\right)\right) & \text { if } \overline{a_{i}}=\varepsilon \\
e_{\otimes} & \text { if } \overline{a_{i}} \neq \varepsilon\end{cases} \\
= & W^{\text {sym }}\left(\left(l_{i-1}, Z_{i-1}, \overline{a_{i-1}}\right),\left(l_{i}, Z_{i}, \overline{a_{i}}\right)\right)
\end{aligned}
$$

Therefore, we have $\mu(\pi)=\mu^{\mathrm{sym}}\left(\pi^{\mathrm{sym}}\right)$.

When $\pi^{\mathrm{sym}} \in \operatorname{ARuns}\left(\mathcal{S}^{\mathrm{sym}}\right)$, we have $l_{0} \in L_{0}, Z_{0}=\mathbf{0}_{C \amalg\{T\}}, \overline{a_{0}}=\varepsilon, l_{n} \in L_{F}$, $\exists \nu_{Z, n} \in Z_{n} . \nu_{Z, n}=|\sigma|$, and $\overline{a_{n}}=\varepsilon$. By Lemma 32, for $\nu_{Z, n} \in Z_{n}$ satisfying $\nu_{Z, n}=|\sigma|$, there is a path $\pi=\left(l_{0}, \nu_{0}, t_{0}, \overline{a_{0}}\right),\left(l_{1}, \nu_{1}, t_{1}, \overline{a_{1}}\right), \ldots,\left(l_{n}, \nu_{n}, t_{n}, \overline{a_{n}}\right)$ of $\mathcal{S}$ such that we have $\left(\nu_{Z, n}\right) \downarrow_{C}=\nu_{n}, \nu_{Z, n}(T)=t_{n} \nu_{0}=\mathbf{0}_{C}, t_{0}=0$, and for any $i \in\{1,2, \ldots, n-1\}$, there exists $\nu_{Z, i} \in Z_{i}$ satisfying $\left(\nu_{Z, i}\right) \downarrow_{C}=\nu_{i}$ and $\nu_{Z, i}(T)=t_{i}$. Therefore $\pi \in A \operatorname{Runs}(\mathcal{S})$ also holds.

Theorem 34. Let $\sigma \in \mathcal{T}\left(\mathbb{D}^{X}\right)$ be a signal and let $\mathcal{W}=(\mathcal{A}, \kappa)$ be a TSWA, where $\mathcal{A}=\left(X, L, L_{0}, L_{F}, C, \Delta, \Lambda\right)$. Let $\mathcal{S}$ and $\mathcal{S}^{\mathrm{sym}}$ be the WTTS and WSTTS of $\sigma$ and $\mathcal{W}$, respectively. If the semiring $\mathbb{S}$ is idempotent, we have $\alpha^{\mathrm{sym}}\left(\mathcal{S}^{\mathrm{sym}}\right) \preceq \alpha(\mathcal{S})$.

Proof. By Lemma 33, there is a mapping $f: \operatorname{ARuns}\left(\mathcal{S}^{\mathrm{sym}}\right) \rightarrow \operatorname{ARuns}(\mathcal{S})$ satisfying $W\left(f\left(\pi^{\mathrm{sym}}\right)\right)=W^{\mathrm{sym}}\left(\pi^{\mathrm{sym}}\right)$. We have

$$
\begin{aligned}
& \alpha^{\mathrm{sym}}\left(\mathcal{S}^{\mathrm{sym}}\right) \oplus \alpha(\mathcal{S}) \\
&=\left(\bigoplus_{\pi^{\mathrm{sym}} \in A \operatorname{Runs}\left(\mathcal{S}^{\mathrm{sym}}\right)} \mu^{\mathrm{sym}}\left(\pi^{\mathrm{sym}}\right)\right) \oplus\left(\bigoplus_{\pi \in A \operatorname{Runs}(\mathcal{S})} \mu(\pi)\right) \\
&=\left(\bigoplus_{\pi \in f\left(\text { ARuns }\left(\mathcal{S}^{\mathrm{sym}}\right)\right)} \mu(\pi)\right) \oplus\left(\bigoplus_{\pi \in A \operatorname{Runs}(\mathcal{S})} \mu(\pi)\right) \\
&\left.\bigoplus_{\pi \in \text { ARns }(\mathcal{S})} \mu(\pi)\right)=\alpha(\mathcal{S})
\end{aligned}
$$

Therefore, we have $\alpha^{\text {sym }}\left(\mathcal{S}^{\text {sym }}\right) \preceq \alpha(\mathcal{S})$.

Proof (Theorem 15). By Theorem 30 and Theorem 34, we have $\alpha(\mathcal{S}) \preceq$ $\alpha^{\text {sym }}\left(\mathcal{S}^{\text {sym }}\right)$. and $\alpha^{\text {sym }}\left(\mathcal{S}^{\text {sym }}\right) \preceq \alpha(\mathcal{S})$. Therefore, we have the following.

$$
\alpha^{\mathrm{sym}}\left(\mathcal{S}^{\mathrm{sym}}\right)=\alpha(\mathcal{S}) \oplus \alpha^{\mathrm{sym}}\left(\mathcal{S}^{\mathrm{sym}}\right)=\alpha^{\mathrm{sym}}\left(\mathcal{S}^{\mathrm{sym}}\right) \oplus \alpha(\mathcal{S})=\alpha(\mathcal{S})
$$




\section{A.3 Proof of Theorem 17}

For locations $l, l^{\prime}$ of $\mathcal{A}=\left(X, L, L_{0}, L_{F}, C, \Delta, \Lambda\right), Z, Z^{\prime} \in \mathcal{Z}(C \amalg\{T\}), \bar{a}, \overline{a^{\prime}} \in$ $\left(\mathbb{D}^{X}\right)^{\circledast}$, and the WSTTS $\mathcal{S}^{\text {sym }}$ of a signal $\sigma$ and $\mathcal{W}=(\mathcal{A}, \kappa)$, We denote the set of paths from $(l, Z, \bar{a})$ to $\left(l^{\prime}, Z^{\prime}, \overline{a^{\prime}}\right)$ of $\mathcal{S}^{\text {sym }}$ as follows.

$$
\begin{aligned}
& \operatorname{Paths}\left(\mathcal{S}^{\mathrm{sym}}, l, Z, \bar{a}, l^{\prime}, Z^{\prime}, \overline{a^{\prime}}\right) \\
= & \left\{\pi^{\mathrm{sym}} \mid(l, Z, \bar{a}), q_{1}^{\mathrm{sym}}, q_{2}^{\mathrm{sym}}, \ldots, q_{n}^{\mathrm{sym}},\left(l^{\prime}, Z^{\prime}, \overline{a^{\prime}}\right) \text { is a path of } \mathcal{S}^{\mathrm{sym}}\right\}
\end{aligned}
$$

By symbolic path value $\mu^{\mathrm{sym}}\left(\pi^{\mathrm{sym}}\right)$, we can rewrite the increment function $\operatorname{incr}(a, t)$ as follows.

$$
\begin{aligned}
& \operatorname{incr}(a, t)(w)=\left\{\left(l^{\prime}, Z^{\prime}, \overline{a^{\prime}}, s^{\prime}\right) \mid l^{\prime} \in L, Z^{\prime} \in \mathcal{Z}(C \amalg\{T\}), \forall \nu^{\prime} \in Z^{\prime} . \nu^{\prime}(T)=t,\right. \\
& \left.\overline{a^{\prime}} \in\left(\mathbb{D}^{X}\right)^{\circledast}, s^{\prime}=\bigoplus_{\substack{(l, Z, \bar{a}, s) \in w \\
\pi^{\mathrm{sym}} \in \operatorname{Paths}\left(\mathcal{S}_{a, t}^{\mathrm{sym}}, l, Z, \bar{a}, l^{\prime}, Z^{\prime}, \overline{a^{\prime}}\right)}} s \otimes \mu^{\mathrm{sym}}\left(\pi^{\mathrm{sym}}\right)\right\}
\end{aligned}
$$

Lemma 35. Let $\mathcal{S}^{\mathrm{sym}}$ be the WSTTS of a signal $\sigma=a_{1}^{\tau_{1}} a_{2}^{\tau_{2}} \cdots a_{n}^{\tau_{n}}$ and a TSWA $\mathcal{W}=(\mathcal{A}, \kappa)$, where $\mathcal{A}=\left(X, L, L_{0}, L_{F}, C, \Delta, \Lambda\right)$. For any $i \in\{1,2, \ldots, n\}$ and for any path

$$
\pi^{\mathrm{sym}}=\left(l_{0},\left\{\mathbf{0}_{C \amalg\{T\}}\right\}, \overline{a_{0}}\right),\left(l_{1}, Z_{1}, \overline{a_{1}}\right), \ldots,\left(l_{m}, Z_{m}, \overline{a_{m}}\right)
$$

of $\mathcal{S}^{\mathrm{sym}}$, if for any $\nu_{m} \in Z_{m}, \nu_{m}(T)=\sum_{k=1}^{i} \tau_{k}$ holds, for any $j \in$ $\{1,2, \ldots, i-1\}$, there exist $l^{\prime} \in L, Z^{\prime} \in \mathcal{Z}(C \amalg\{T\})$, and $\bar{a}^{\prime} \in$ $\left(\mathbb{D}^{X}\right)^{\circledast}$ such that for any $\nu^{\prime} \in Z^{\prime}$, we have $\nu^{\prime}(T)=\sum_{k=1}^{j} \tau_{k}$, and there are paths $\pi^{\mathrm{sym} \prime} \in \operatorname{Paths}\left(\mathcal{S}^{\mathrm{sym}}, l_{0},\left\{\mathbf{0}_{C \amalg\{T\}}\right\}, \overline{a_{0}}, l^{\prime}, Z^{\prime}, \overline{a^{\prime}}\right)$ and $\pi^{\mathrm{sym} \prime \prime} \in$ $\operatorname{Paths}\left(\mathcal{S}^{\mathrm{sym}}, l^{\prime}, Z^{\prime}, \overline{a^{\prime}}, l_{m}, Z_{m}, \overline{a_{m}}\right) \quad$ satisfying $\mu^{\mathrm{sym}}\left(\pi^{\mathrm{sym}}\right)=\mu^{\mathrm{sym}}\left(\pi^{\mathrm{sym} \prime}\right) \otimes$ $\mu^{\mathrm{sym}}\left(\pi^{\mathrm{sym} \prime \prime}\right)$.

Proof. If there exists $h \in\{1,2, \ldots, m\}$ such that for any $\nu_{h} \in Z_{h}$, we have $\nu_{h}(T)=\sum_{k=1}^{j} \tau_{k}$, we have the following.

$$
\begin{aligned}
\pi^{\mathrm{sym} \prime} & =\left(l_{0},\left\{\mathbf{0}_{C \amalg\{T\}}\right\}, \overline{a_{0}}\right),\left(l_{1}, Z_{1}, \overline{a_{1}}\right), \ldots,\left(l_{h}, Z_{h}, \overline{a_{h}}\right) \\
& \in \operatorname{Paths}\left(\mathcal{S}^{\mathrm{sym}}, l_{0},\left\{\mathbf{0}_{C \amalg\{T\}}\right\}, \overline{a_{0}}, l^{\prime}, Z^{\prime}, \overline{a^{\prime}}\right) \\
\pi^{\mathrm{sym} \prime \prime} & =\left(l_{h}, Z_{h}, \overline{a_{h}}\right),\left(l_{h+1}, Z_{h+1}, \overline{a_{h+1}}\right), \ldots,\left(l_{m}, Z_{m}, \overline{a_{m}}\right) \\
& \in \operatorname{Paths}\left(\mathcal{S}^{\mathrm{sym}}, l^{\prime}, Z^{\prime}, \overline{a^{\prime}}, l_{m}, Z_{m}, \overline{a_{m}}\right) \\
\mu^{\mathrm{sym}}\left(\pi^{\mathrm{sym}}\right) & =\mu^{\mathrm{sym}}\left(\pi^{\mathrm{sym} \prime}\right) \otimes \mu^{\mathrm{sym}}\left(\pi^{\mathrm{sym} \prime \prime}\right)
\end{aligned}
$$

Assume for any $h \in\{1,2, \ldots, m\}$, there is $\nu_{h} \in Z_{h}$ satisfying $\nu_{h}(T) \neq$ $\sum_{k=1}^{j} \tau_{k}$. Let $h \in\{1,2, \ldots, m-1\}$ such that for any $\nu_{h} \in Z_{h}$, we have $\nu_{h}(T)<\sum_{k=1}^{j} \tau_{k}$ and for any $\nu_{h+1} \in Z_{h+1}$, we have $\nu_{h+1}(T)>\sum_{k=1}^{j} \tau_{k}$. Since $\left(\left(l_{h}, Z_{h}, \overline{a_{h}}\right),\left(l_{h+1}, Z_{h+1}, \overline{a_{h+1}}\right)\right) \in \rightarrow^{\text {sym }}$, there is $g \in\{1,2, \ldots, n\}$, satisfying $l_{h}=l_{h+1}, \overline{a_{h+1}}=\overline{a_{h}} \circ \operatorname{Values}\left(\sigma\left(\left[\nu_{h}(T), \nu_{h+1}(T)\right)\right)\right)$, and $Z_{h+1}=\{\nu+\tau \mid$ 
$\left.\nu \in Z, \tau \in \mathbb{R}_{>0}\right\} \cap M_{g}$, where $\nu_{h} \in Z_{h}, \nu_{h+1} \in Z_{h+1}$ and $M_{g}=\left\{\nu \mid \sum_{k=0}^{g-1} \tau_{k}<\right.$ $\left.\nu(T)<\sum_{k=0}^{g} \tau_{k}\right\}$. Let $Z^{\prime}=\left\{\nu+\tau \mid \nu \in Z, \tau \in \mathbb{R}_{>0}\right\} \cap\left\{\nu \mid \sum_{k=0}^{j} \tau_{k}=\nu(T)\right\}$ and $\overline{a^{\prime}}=\overline{a_{h}} \circ \operatorname{Values}\left(\sigma\left(\left[\nu_{h}(T), \sum_{k=0}^{j} \tau_{k}\right)\right)\right)$. Since $\left(\left(l_{h}, Z_{h}, \overline{a_{h}}\right),\left(l_{h}, Z^{\prime}, \overline{a^{\prime}}\right)\right) \in \rightarrow^{\text {sym }}$ and $\left(\left(l_{h+1}, Z^{\prime}, \overline{a^{\prime}}\right),\left(l_{h+1}, Z_{h+1}, \overline{a_{h+1}}\right)\right) \in \rightarrow^{\text {sym }}$ hold, we have the following.

$$
\begin{aligned}
\pi^{\mathrm{sym} \prime} & =\left(l_{0},\left\{\mathbf{0}_{C \amalg\{T\}}\right\}, \overline{a_{0}}\right),\left(l_{1}, Z_{1}, \overline{a_{1}}\right), \ldots,\left(l_{h}, Z_{h}, \overline{a_{h}}\right),\left(l_{h}, Z^{\prime}, \overline{a^{\prime}}\right) \\
& \in \operatorname{Paths}\left(\mathcal{S}^{\mathrm{sym}}, l_{0},\left\{\mathbf{0}_{C \amalg\{T\}}\right\}, \overline{a_{0}}, l_{h^{\prime}}, Z^{\prime}, \overline{a^{\prime}}\right) * \\
\pi^{\mathrm{sym} \prime \prime} & =\left(l_{h+1}, Z^{\prime}, \overline{a^{\prime}}\right),\left(l_{h+1}, Z_{h+1}, \overline{a_{h+1}}\right), \ldots,\left(l_{m}, Z_{m}, \overline{a_{m}}\right) \\
& \in P a t h s\left(\mathcal{S}^{\mathrm{sym}}, l_{h+1}, Z^{\prime}, \overline{a^{\prime}}, l_{m}, Z_{m}, \overline{a_{m}}\right) \\
\mu^{\mathrm{sym}}\left(\pi^{\mathrm{sym}}\right) & =\mu^{\mathrm{sym}}\left(\pi^{\mathrm{sym} \prime}\right) \otimes \mu^{\mathrm{sym}}\left(\pi^{\mathrm{sym} \prime \prime}\right)
\end{aligned}
$$

Lemma 36. For a TSWA $\mathcal{W}$, a signal $\sigma=a_{1}^{\tau_{1}} a_{2}^{\tau_{2}} \cdots a_{n}^{\tau_{n}}$, and $i \in\{1,2, \ldots, n\}$, we have the following.

$$
\begin{gathered}
\text { weight }_{i}=\left\{\left(l_{i}, Z_{i}, \overline{a_{i}}, s_{i}\right) \mid l_{i} \in L, Z_{i} \in \mathcal{Z}(C \amalg\{T\}), \overline{a_{i}} \in\left(\mathbb{D}^{X}\right)^{\circledast},\right. \\
s_{i}=\bigoplus_{\pi^{\mathrm{sym}} \in \operatorname{Paths}\left(\mathcal{S}^{\mathrm{sym}}, l_{0}, \mathbf{0}_{C} C \amalg\{T\}, \varepsilon, l_{i}, Z_{i}, \overline{a_{i}}\right)} \mu^{\mathrm{sym}}\left(\pi^{\mathrm{sym}}\right) \\
\left.\forall \nu^{\prime} \in Z^{\prime} \cdot \nu^{\prime}(T)=\sum_{j=1}^{i} \tau_{j}\right\}
\end{gathered}
$$

Proof. We prove by induction on $i$. If $i=1$, we have the following.

$$
\begin{aligned}
& \text { weight }_{1}=\operatorname{incr}\left(a_{1}, \tau_{1}\right)\left(\left\{\left(l_{0}, \mathbf{0}_{C \amalg\{T\}}, \varepsilon, e_{\otimes}\right) \mid l_{0} \in L_{0}\right\}\right) \\
& =\left\{\left(l^{\prime}, Z^{\prime}, \overline{a^{\prime}}, s^{\prime}\right) \mid l^{\prime} \in L, Z^{\prime} \in \mathcal{Z}(C \amalg\{T\}), \overline{a^{\prime}} \in\left(\mathbb{D}^{X}\right)^{\circledast},\right.
\end{aligned}
$$

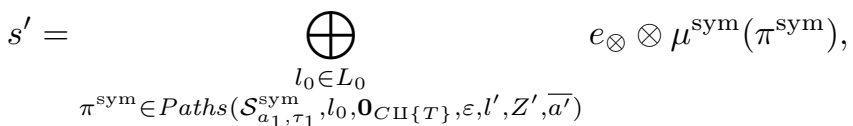

$$
\begin{aligned}
& \left.\forall \nu^{\prime} \in Z^{\prime} \cdot \nu^{\prime}(T)=\tau_{1}\right\} \\
& =\left\{\left(l^{\prime}, Z^{\prime}, \overline{a^{\prime}}, s^{\prime}\right) \mid l^{\prime} \in L, Z^{\prime} \in \mathcal{Z}(C \amalg\{T\}), \overline{a^{\prime}} \in\left(\mathbb{D}^{X}\right)^{\circledast},\right. \\
& s^{\prime}=\underset{\substack{l_{0} \in L_{0} \\
\pi^{\mathrm{sym}} \in \operatorname{Paths}\left(\mathcal{S}^{\mathrm{sym}}, l_{0}, \mathbf{0}_{C \amalg\{T\}}, \varepsilon, l^{\prime}, Z^{\prime}, \overline{a^{\prime}}\right)}}{ } e_{\otimes} \otimes \mu^{\mathrm{sym}}\left(\pi^{\mathrm{sym}}\right), \\
& \left.\forall \nu^{\prime} \in Z^{\prime} \cdot \nu^{\prime}(T)=\tau_{1}\right\}
\end{aligned}
$$


If $i>1$, by induction hypothesis, we have the following.

$$
\begin{aligned}
& \text { weight }_{i}=\operatorname{incr}\left(a_{i}, \tau_{i}\right)\left(\text { weight }_{i-1}\right) \\
& =\left\{\left(l^{\prime}, Z^{\prime}, \overline{a^{\prime}}, s^{\prime}\right) \mid l^{\prime} \in L, Z^{\prime} \in \mathcal{Z}(C \amalg\{T\}), \overline{a^{\prime}} \in\left(\mathbb{D}^{X}\right)^{\circledast},\right. \\
& s^{\prime}=\bigoplus s \otimes \mu^{\mathrm{sym}}\left(\pi^{\mathrm{sym}}\right), \\
& (l, Z, \bar{a}, s) \in \text { weight }_{i-1} \\
& \pi^{\mathrm{sym}} \in \operatorname{Paths}\left(\mathcal{S}_{a_{i}, \tau_{i}}^{\mathrm{sym}}, l, Z, \bar{a}, l^{\prime}, Z^{\prime}, \overline{a^{\prime}}\right) \\
& \left.\forall \nu^{\prime} \in Z^{\prime} . \nu^{\prime}(T)=\sum_{j=1}^{i} \tau_{j}\right\} \\
& =\left\{\left(l^{\prime}, Z^{\prime}, \overline{a^{\prime}}, s^{\prime}\right) \mid l^{\prime} \in L, Z^{\prime} \in \mathcal{Z}(C \amalg\{T\}), \overline{a^{\prime}} \in\left(\mathbb{D}^{X}\right)^{\circledast},\right. \\
& s^{\prime}=\bigoplus_{l_{0} \in L_{0}, l \in L, Z \in \mathcal{Z}(C \amalg\{T\}), \bar{a} \in\left(\mathbb{D}^{X}\right)^{\circledast},} \mu^{\mathrm{sym}}\left(\pi^{\mathrm{sym} \prime}\right) \otimes \mu^{\mathrm{sym}}\left(\pi^{\mathrm{sym}}\right), \\
& \pi^{\mathrm{sym}} \in \operatorname{Paths}\left(\mathcal{S}_{a_{i}, \tau_{i}}^{\mathrm{sym}}, l, Z, \bar{a}, l^{\prime}, Z^{\prime}, \overline{a^{\prime}}\right) \\
& \pi^{\mathrm{sym} \prime} \in \operatorname{Paths}\left(\mathcal{S}^{\mathrm{sym}}, l_{0}, \mathbf{0}_{C \amalg\{T\}}, \varepsilon, l, Z, \bar{a}\right) \\
& \forall \nu \in Z . \nu(T)=\sum_{j=1}^{i-1} \tau_{j} \\
& \left.\forall \nu^{\prime} \in Z^{\prime} . \nu^{\prime}(T)=\sum_{j=1}^{i} \tau_{j}\right\} \\
& =\left\{\left(l^{\prime}, Z^{\prime}, \overline{a^{\prime}}, s^{\prime}\right) \mid l^{\prime} \in L, Z^{\prime} \in \mathcal{Z}(C \amalg\{T\}), \overline{a^{\prime}} \in\left(\mathbb{D}^{X}\right)^{\circledast},\right. \\
& s^{\prime}=\underset{\substack{l_{0} \in L_{0} \\
\pi^{\mathrm{sym}} \in \operatorname{Paths}\left(\mathcal{S}^{\mathrm{sym}}, l_{0}, \mathbf{0}_{C \amalg\{T\}}, \varepsilon, l^{\prime}, Z^{\prime}, \overline{a^{\prime}}\right)}}{ } \mu^{\mathrm{sym}}\left(\pi^{\mathrm{sym}}\right), \\
& \left.\forall \nu^{\prime} \in Z^{\prime} \cdot \nu^{\prime}(T)=\sum_{j=1}^{i} \tau_{j}\right\}
\end{aligned}
$$

Proof (Theorem 17). By Lemma 36, we have the following.

$$
\begin{aligned}
& \bigoplus_{\text {sym }} s \\
& (l, Z, \bar{a}) \in Q_{F}^{\mathrm{sym}} \\
& (l, Z, \bar{a}, s) \in \text { weight }_{n} \\
& =\bigoplus_{\begin{array}{c}
(l, Z, \bar{a}) \in Q_{0}^{\mathrm{sym}} \\
\left(l^{\prime}, Z^{\prime}, \bar{a}^{\prime}\right) \in Q_{F}^{\mathrm{sym}} \\
\pi^{\mathrm{sym}} \in \operatorname{Paths}\left(\mathcal{S}^{\mathrm{sym}}, l, Z, \bar{a}, l^{\prime}, Z^{\prime}, \bar{a}^{\prime}\right)
\end{array}} \mu^{\mathrm{sym}}\left(\pi^{\mathrm{sym}}\right) \\
& =\bigoplus_{\pi^{\mathrm{sym}} \in A \operatorname{Runs}\left(\mathcal{S}^{\mathrm{sym}}\right)} \mu^{\mathrm{sym}}\left(\pi^{\mathrm{sym}}\right) \\
& =\alpha^{\mathrm{sym}}\left(\mathcal{S}^{\mathrm{sym}}\right)
\end{aligned}
$$


Table 2: Execution time and memory usage under long signals for OvERshoot and Ringing for sup-inf semiring

\begin{tabular}{ccccc}
\hline$|\sigma|$ & $\begin{array}{c}\text { Execution Time [s] } \\
\text { (Overshoot) }\end{array}$ & $\begin{array}{c}\text { Memory Usage [KiB] } \\
\text { (Overshoot) }\end{array}$ & $\begin{array}{c}\text { Execution Time [s] } \\
\text { (Ringing) }\end{array}$ & $\begin{array}{c}\text { Memory Usage [KiB] } \\
\text { (Ringing) }\end{array}$ \\
\hline 60,000 & 1.59 & $7,196.20$ & 13.06 & $7,949.20$ \\
120,000 & 3.19 & $7,143.60$ & 26.17 & $7,924.00$ \\
180,000 & 4.85 & $7,197.80$ & 39.03 & $7,960.00$ \\
240,000 & 6.44 & $7,160.60$ & 52.06 & $7,977.60$ \\
300,000 & 8.07 & $7,165.60$ & 65.19 & $7,912.20$ \\
360,000 & 9.71 & $7,147.20$ & 78.13 & $7,953.60$ \\
420,000 & 11.40 & $7,197.80$ & 91.37 & $7,961.20$ \\
480,000 & 13.01 & $7,195.60$ & 104.12 & $7,933.20$ \\
540,000 & 14.60 & $7,160.00$ & 117.49 & $7,966.40$ \\
600,000 & 16.28 & $7,202.80$ & 131.00 & \\
\hline
\end{tabular}

Table 3: Execution time and memory usage under long signals for OvERSHOoT (UNBOUNDED) for sup-inf semiring

\begin{tabular}{ccc}
\hline$|\sigma|$ & Execution Time $[\mathrm{s}]$ & Memory Usage $[\mathrm{KiB}]$ \\
\hline 1,000 & 0.14 & $9,050.60$ \\
2,000 & 0.92 & $14,633.80$ \\
3,000 & 2.97 & $23,667.80$ \\
4,000 & 6.79 & $36,452.00$ \\
5,000 & 13.46 & $52,806.80$ \\
6,000 & 23.22 & $72,639.40$ \\
7,000 & 36.86 & $96,220.80$ \\
8,000 & 55.63 & $123,263.00$ \\
9,000 & 79.23 & $153,900.00$ \\
10,000 & 112.15 & $188,054.00$ \\
\hline
\end{tabular}

\section{B Performance comparison between the benchmarks}

In Fig. 9, we observe that the execution time and memory usage of Ringing are higher than those of OvERSHOOT. This is because the TSA of RINGING of is more complex than that of OvERSHOOT: it has more states and clock variables, and it contains a loop. We also observe that for RINGING, the execution time for the tropical semiring is shorter. This is because staying at the locations with $T$ minimizes the weight for tropical semiring, and we need less exploration.

\section{Detailed experimental results}

The detailed experimental results are summarized in Tables 2 to 7 . 
Table 4: Execution time and memory usage under high frequency for OverSHOOT and RINGING for sup-inf semiring

\begin{tabular}{ccccc}
\hline Sampling Freq. [Hz] & $\begin{array}{c}\text { Execution Time [s] } \\
\text { (Overshoot) }\end{array}$ & $\begin{array}{c}\text { Memory Usage [KiB] } \\
\text { (Overshoot) }\end{array}$ & $\begin{array}{c}\text { Execution Time [s] } \\
\text { (RINGING) }\end{array}$ & $\begin{array}{c}\text { Memory Usage [KiB] } \\
\text { (RINGing) }\end{array}$ \\
\hline 0.1 & $1.60 \cdot 10^{0}$ & $7,169.4$ & $1.30 \cdot 10^{1}$ & 7,943 \\
0.2 & $4.85 \cdot 10^{0}$ & 7,518 & $4.56 \cdot 10^{1}$ & $9,906.2$ \\
0.3 & $9.93 \cdot 10^{0}$ & $8,234.8$ & $1.10 \cdot 10^{2}$ & $14,688.8$ \\
0.4 & $1.64 \cdot 10^{1}$ & 9,277 & $2.22 \cdot 10^{2}$ & 18,209 \\
0.5 & $2.53 \cdot 10^{1}$ & 10,723 & $3.78 \cdot 10^{2}$ & $25,469.2$ \\
0.6 & $3.79 \cdot 10^{1}$ & $13,019.6$ & $6.35 \cdot 10^{2}$ & $36,310.2$ \\
0.7 & $5.35 \cdot 10^{1}$ & $16,250.6$ & $9.41 \cdot 10^{2}$ & $50,715.4$ \\
0.8 & $7.10 \cdot 10^{1}$ & $20,134.8$ & $1.42 \cdot 10^{3}$ & $56,401.8$ \\
0.9 & $9.66 \cdot 10^{1}$ & 25,429 & $1.98 \cdot 10^{3}$ & 80,871 \\
1.0 & $1.22 \cdot 10^{2}$ & $31,358.6$ & $2.57 \cdot 10^{3}$ & $91,547.2$ \\
\hline
\end{tabular}

Table 5: Execution time and memory usage under long signals for Overshoot and Ringing for tropical semiring

\begin{tabular}{ccccc}
\hline$|\sigma|$ & $\begin{array}{c}\text { Execution Time [s] } \\
\text { (Overshoot) }\end{array}$ & $\begin{array}{c}\text { Memory Usage [KiB] } \\
\text { (Overshoot) }\end{array}$ & $\begin{array}{c}\text { Execution Time [s] } \\
\text { (Ringing) }\end{array}$ & $\begin{array}{c}\text { Memory Usage [KiB] } \\
\text { (Ringing) }\end{array}$ \\
\hline 60,000 & 1.92 & $7,292.80$ & 6.67 & $7,678.80$ \\
120,000 & 3.86 & $7,273.00$ & 13.17 & $7,680.00$ \\
180,000 & 5.83 & $7,278.00$ & 19.79 & $7,710.80$ \\
240,000 & 7.76 & $7,315.40$ & 26.30 & $7,704.00$ \\
300,000 & 9.75 & $7,304.80$ & 32.88 & $7,703.20$ \\
360,000 & 11.69 & $7,287.80$ & 39.35 & $7,709.40$ \\
420,000 & 13.67 & $7,288.00$ & 46.11 & $7,712.00$ \\
480,000 & 15.64 & $7,281.80$ & 52.73 & $7,737.20$ \\
540,000 & 17.57 & $7,283.40$ & 59.20 & $7,728.00$ \\
600,000 & 19.48 & $7,266.00$ & 65.67 & $7,737.60$ \\
\hline
\end{tabular}

Table 6: Execution time and memory usage under long signals for Overshoot (UnBounded) for tropical semiring

\begin{tabular}{ccc}
\hline$|\sigma|$ & Execution Time $[\mathrm{s}]$ & Memory Usage $[\mathrm{KiB}]$ \\
\hline 1,000 & 0.18 & $9,799.40$ \\
2,000 & 1.16 & $16,840.40$ \\
3,000 & 3.59 & $28,256.20$ \\
4,000 & 8.22 & $44,139.40$ \\
5,000 & 15.75 & $64,509.40$ \\
6,000 & 27.34 & $89,218.40$ \\
7,000 & 43.33 & $118,498.00$ \\
8,000 & 64.29 & $152,075.00$ \\
9,000 & 90.74 & $190,065.00$ \\
10,000 & 122.87 & $232,474.00$ \\
\hline
\end{tabular}


Table 7: Execution time and memory usage under high frequency for OverSHOOT and RINGING for tropical semiring

\begin{tabular}{ccccc}
\hline Sampling Freq. [Hz] & $\begin{array}{c}\text { Execution Time [s] } \\
\text { (Overshoot) }\end{array}$ & $\begin{array}{c}\text { Memory Usage [KiB] } \\
\text { (Overshoot) }\end{array}$ & $\begin{array}{c}\text { Execution Time [s] } \\
\text { (RINGing) }\end{array}$ & $\begin{array}{c}\text { Memory Usage [KiB] } \\
\text { (Ringing) }\end{array}$ \\
\hline 0.1 & $1.91 \cdot 10^{0}$ & $7,274.2$ & $6.66 \cdot 10^{0}$ & $7,646.4$ \\
0.2 & $6.94 \cdot 10^{0}$ & $7,704.8$ & $1.17 \cdot 10^{1}$ & $7,910.2$ \\
0.3 & $1.46 \cdot 10^{1}$ & $8,547.2$ & $1.66 \cdot 10^{1}$ & $8,302.6$ \\
0.4 & $2.44 \cdot 10^{1}$ & $9,836.8$ & $2.17 \cdot 10^{1}$ & $8,666.2$ \\
0.5 & $3.80 \cdot 10^{1}$ & $11,905.8$ & $2.88 \cdot 10^{1}$ & $8,855.8$ \\
0.6 & $5.75 \cdot 10^{1}$ & $14,764.2$ & $3.29 \cdot 10^{1}$ & $9,730.8$ \\
0.7 & $8.17 \cdot 10^{1}$ & $18,501.6$ & $4.14 \cdot 10^{1}$ & $9,831.8$ \\
0.8 & $1.10 \cdot 10^{2}$ & 23,484 & $5.23 \cdot 10^{1}$ & 10,307 \\
0.9 & $1.50 \cdot 10^{2}$ & $29,905.4$ & $6.36 \cdot 10^{1}$ & $11,291.2$ \\
1.0 & $1.97 \cdot 10^{2}$ & $37,465.2$ & $6.70 \cdot 10^{1}$ & $11,443.8$ \\
\hline
\end{tabular}

